\title{
On the convergence of solutions of variational problems with variable implicit pointwise constraints in variable domains
}

\author{
Alexander A. Kovalevsky ${ }^{1,2}$ \\ Received: 14 July 2018 / Accepted: 19 November 2018 / Published online: 1 December 2018 \\ (c) Fondazione Annali di Matematica Pura ed Applicata and Springer-Verlag GmbH Germany, part of Springer \\ Nature 2018
}

\begin{abstract}
In this paper, we give sufficient conditions for the convergence of minimizers and minimum values of integral and more general functionals $J_{s}: W^{1, p}\left(\Omega_{s}\right) \rightarrow \mathbb{R}$ on the sets $U_{s}\left(h_{s}\right)=$ $\left\{v \in W^{1, p}\left(\Omega_{s}\right): h_{s}(v) \leqslant 0\right.$ a.e. in $\left.\Omega_{s}\right\}$, where $p>1,\left\{\Omega_{s}\right\}$ is a sequence of domains contained in a bounded domain $\Omega$ of $\mathbb{R}^{n}(n \geqslant 2)$, and $\left\{h_{s}\right\}$ is a sequence of functions on $\mathbb{R}$. In so doing, we assume that the considered functionals $\Gamma$-converge to a functional defined on $W^{1, p}(\Omega)$ and the spaces $W^{1, p}\left(\Omega_{s}\right)$ are strongly connected with the space $W^{1, p}(\Omega)$. Certain conditions on the relation between the functions $h_{s}$ and a function $h: \mathbb{R} \rightarrow \mathbb{R}$ are also required in our main results.
\end{abstract}

Keywords Integral functional - Variational problem · Implicit pointwise constraints ·

Minimizer $\cdot$ Minimum value $\cdot \Gamma$-Convergence $\cdot$ Variable domains

Mathematics Subject Classification 49J40 · 49J45

\section{Introduction}

In this paper, for a given bounded domain $\Omega$ of $\mathbb{R}^{n}(n \geqslant 2)$ and a given sequence of domains $\Omega_{s}$ contained in $\Omega$, we consider a sequence of functionals $J_{s}: W^{1, p}\left(\Omega_{s}\right) \rightarrow \mathbb{R}$ of the structure $J_{s}=F_{s}+G_{s}$, where $p>1,\left\{F_{s}\right\}$ is a sequence of integral functionals whose integrands satisfy certain convexity and growth conditions, and $\left\{G_{s}\right\}$ is a sequence of weakly continuous functionals. Along with this, we consider the sequence of sets

$$
U_{s}\left(h_{s}\right)=\left\{v \in W^{1, p}\left(\Omega_{s}\right): h_{s}(v) \leqslant 0 \text { a.e. in } \Omega_{s}\right\}
$$

$\bowtie \quad$ Alexander A. Kovalevsky

alexkv171@mail.ru

1 Krasovskii Institute of Mathematics and Mechanics, The Ural Branch of the Russian Academy of Sciences, Sofia Kovalevskaya St. 16, Yekaterinburg, Russia 620990

2 Institute of Natural Sciences and Mathematics, Ural Federal University, pr. Lenina 51, Yekaterinburg, Russia 620000 
where $\left\{h_{s}\right\}$ is a sequence of functions on $\mathbb{R}$. We are interested in conditions for the convergence of minimizers and minimum values of the functionals $J_{s}$ on the sets $U_{s}\left(h_{s}\right)$ to a minimizer and the minimum value of a limit functional on a limit set.

It is easy to see that the variational problems under consideration include but, in general, are not reduced to standard unilateral and bilateral problems with constraints of the forms $v \geqslant \varphi_{s}, v \leqslant \psi_{s}$, and $\varphi_{s} \leqslant v \leqslant \psi_{s}$ a.e. in $\Omega_{s}$, where $\left\{\varphi_{s}\right\},\left\{\psi_{s}\right\} \subset \mathbb{R}$.

In this connection, we note that the convergence of solutions of variational problems with constraints of the forms $v \geqslant \varphi, v \leqslant \psi$, and $\varphi \leqslant v \leqslant \psi$ a.e. in $\Omega_{s}$, where $\varphi, \psi \in W^{1, p}(\Omega)$, was studied, for instance, in [11,17]. In these works, the conditions for the convergence of solutions of the mentioned problems include the requirement of $\Gamma$-convergence of the corresponding functionals to a functional defined on the space $W^{1, p}(\Omega)$ and the requirement of a certain (called strong) connectedness of the spaces $W^{1, p}\left(\Omega_{S}\right)$ with the space $W^{1, p}(\Omega)$.

Similarly, in the present paper, in particular, the $\Gamma$-convergence of the sequence $\left\{F_{s}\right\}$ to a functional $F: W^{1, p}(\Omega) \rightarrow \mathbb{R}$, a certain convergence of the sequence $\left\{G_{s}\right\}$ to a functional $G: W^{1, p}(\Omega) \rightarrow \mathbb{R}$, and the strong connectedness of the spaces $W^{1, p}\left(\Omega_{S}\right)$ with the space $W^{1, p}(\Omega)$ are required to prove the convergence of minimizers and minimum values of the functionals $J_{s}$ on the sets $U_{s}\left(h_{s}\right)$. At the same time, we assume that, for every $s \in \mathbb{N}$, the integrand $f_{s}: \Omega_{s} \times \mathbb{R}^{n} \rightarrow \mathbb{R}$ of the functional $F_{s}$ satisfies the inequality

$$
c_{1}|\xi|^{p}-\mu_{s}(x) \leqslant f_{s}(x, \xi) \leqslant c_{2}|\xi|^{p}+\mu_{s}(x)
$$

for almost every $x \in \Omega_{s}$ and every $\xi \in \mathbb{R}^{n}$, where $c_{1}$ and $c_{2}$ are preassigned positive constants and $\mu_{s} \in L^{1}\left(\Omega_{s}\right), \mu_{s} \geqslant 0$ in $\Omega_{s}$. The only assumption on the domain $\Omega$ is that the embedding of $W^{1, p}(\Omega)$ into $L^{p}(\Omega)$ is compact. Moreover, in our main results (see Theorems 4.14.3), we require certain conditions on the relation between the functions $h_{s}$ and a function $h: \mathbb{R} \rightarrow \mathbb{R}$. Actually, these conditions relate the sets $\Phi\left(h_{s}\right)=\left\{t \in \mathbb{R}: h_{s}(t) \leqslant 0\right\}$ to the set $\Phi(h)=\{t \in \mathbb{R}: h(t) \leqslant 0\}$. In particular, the assumed conditions imply the convergence of the sequence $\left\{\Phi\left(h_{s}\right)\right\}$ to the set $\Phi(h)$ in the sense of Kuratowski [21, Sect. 29], and the sets $\Phi\left(h_{S}\right)$ are assumed to be nonempty and closed. The convexity of the sets $\Phi\left(h_{s}\right)$ is not required. The obtained results say that minimizers and minimum values of the functionals $J_{S}$ on the sets $U_{s}\left(h_{s}\right)$ converge (along a subsequence) to a minimizer and the minimum value of the functional $F+G$ on the set $U(h)=\left\{v \in W^{1, p}(\Omega): h(v) \leqslant 0\right.$ a.e. in $\left.\Omega\right\}$.

We note that the sets $U_{s}\left(h_{s}\right)$ originally defined by (1.1) can be written as follows:

$$
U_{s}\left(h_{s}\right)=\left\{v \in W^{1, p}\left(\Omega_{s}\right): v(x) \in \Phi\left(h_{s}\right) \text { for a.e. } x \in \Omega_{s}\right\} .
$$

On the other hand, if we have a sequence of sets $\Psi_{s} \subset \mathbb{R}$ and, for every $s \in \mathbb{N}$,

$$
V_{s}=\left\{v \in W^{1, p}\left(\Omega_{s}\right): v(x) \in \Psi_{s} \text { for a.e. } x \in \Omega_{s}\right\},
$$

then the sets $V_{s}$ can be represented as $V_{s}=U_{s}\left(h_{s}\right)$, where functions $h_{s}: \mathbb{R} \rightarrow \mathbb{R}$ are defined, for instance, by $h_{s}(t)=0$ if $t \in \Psi_{s}$ and $h_{s}(t)=1$ if $t \notin \Psi_{s}$. Obviously, for these functions, we have $\Phi\left(h_{s}\right)=\Psi_{s}$ for every $s \in \mathbb{N}$.

Thus, essentially, our results concern the convergence of minimizers and minimum values of the functionals $J_{S}$ on sets of the form (1.3) without the assumption of convexity of the sets $\Psi_{s}$. To the best of our knowledge, this case has almost not been studied even for the domains $\Omega_{s}$ coinciding with the domain $\Omega$. The only exceptions are our recent works $[18,19]$. In the short note [18], we only announced Theorems 4.1 and 4.2 of the present paper. In [19], we studied in detail the convergence of minimizers and minimum values of functionals on sets of the form (1.1) defined by rapidly oscillating periodic functions $h_{s}$. This special case is not covered by the main results of the present paper (see Remark 4.6). 
As for the notion of strong connectedness of a sequence of Sobolev spaces and the notion of $\Gamma$-convergence of functionals with variable domain of definition used in the present paper, additionally to $[11,17]$, we refer the reader, for instance, to $[13,15]$. We remark that the notion of strong connectedness of Sobolev spaces goes back to [10], where the condition of strong connectedness of $n$-dimensional domains was introduced. This condition can be considered as a prototype of the mentioned notion of strong connectedness of Sobolev spaces.

Concerning the notion of $\Gamma$-convergence of functionals with the same domain of definition and related results, see, for instance, [5,8,26,27]. With the use of the techniques of $\Gamma$-convergence theory, the convergence of minimizers and minimum values of variational problems with general varying unilateral obstacles in a fixed domain was studied in [4] for integral functionals whose integrands satisfy a uniform growth and coercivity condition. A similar question related to variational problems with general varying bilateral obstacles for a quadratic integral functional was investigated in [3]. For results on the convergence of solutions of variational inequalities with $G$-convergent operators and unilateral and bilateral constraints in different cases, we refer the reader, for instance, to [2,6,14,23]. The unilateral and bilateral constraints considered in the mentioned papers can be written in the form

$$
v(x) \in \Psi_{s}(x)
$$

for a.e. $x$ in the corresponding domain, where $\Psi_{s}(x)$ are closed convex sets in $\mathbb{R}$. The same also concerns the variational problems with implicit pointwise constraints studied in [20] for integral functionals on weighted Sobolev spaces associated with the domains $\Omega_{s}$. More exactly, the implicit constraints considered in [20] are reduced to the form (1.4) with $\Psi_{s}(x)=$ $[\varphi(x),+\infty)$, where $\varphi: \Omega \rightarrow \overline{\mathbb{R}}$, and $\Psi_{s}(x)=\left[\alpha_{-}, \alpha_{+}\right] \cap \mathbb{R}$, where $\alpha_{-} \in[-\infty, 0]$ and $\alpha_{+} \in[0,+\infty]$. The asymptotic behavior of a sequence of minimization problems for an integral functional on a Sobolev space of vector valued functions was studied in [7] in the case of constraints of the form (1.4) with closed convex sets $\Psi_{s}(x)$ in $\mathbb{R}^{k}(k \geqslant 1)$.

The present paper is organized as follows. In Sect. 2, we give necessary assumptions and definitions. In Sect. 3, we prove a number of useful auxiliary propositions. In Sect. 4, we state and prove our main Theorems 4.1-4.3. The difference in their formulations is the difference in both the assumptions on the functions $\mu_{s}$ that appear in (1.2) and the conditions on the above sets $\Phi\left(h_{s}\right)$ and $\Phi(h)$. Thus, in Theorem 4.1, we assume that, for every sequence of measurable sets $K_{s} \subset \Omega_{s}$ such that meas $K_{s} \rightarrow 0$, the integrals of the functions $\mu_{s}$ over the sets $K_{s}$ tend to zero, while, in Theorems 4.2 and 4.3, we assume the stronger condition $\left\|\mu_{s}\right\|_{L^{1}\left(\Omega_{s}\right)} \rightarrow 0$. Finally, in Sect. 5, we give comments and examples on the made assumptions and the conditions of Theorems 4.1-4.3. A special attention is paid to the construction of examples justifying the importance of the conditions on the sets $\Phi\left(h_{s}\right)$ and $\Phi(h)$ in Theorems 4.1-4.3 and of the condition $\left\|\mu_{s}\right\|_{L^{1}\left(\Omega_{s}\right)} \rightarrow 0$ in Theorems 4.2 and 4.3.

\section{Assumptions and definitions}

Let $n \in \mathbb{N}, n \geqslant 2$, let $\Omega$ be a bounded domain of $\mathbb{R}^{n}$, and let $p>1$. Let $\left\{\Omega_{s}\right\}$ be a sequence of domains of $\mathbb{R}^{n}$ contained in $\Omega$.

It is easy to see that if $v \in W^{1, p}(\Omega)$ and $s \in \mathbb{N}$, then $\left.v\right|_{\Omega_{s}} \in W^{1, p}\left(\Omega_{s}\right)$.

Definition 2.1 If $s \in \mathbb{N}$, then $q_{s}: W^{1, p}(\Omega) \rightarrow W^{1, p}\left(\Omega_{s}\right)$ is the mapping such that, for every function $v \in W^{1, p}(\Omega)$, we have $q_{s} v=\left.v\right|_{\Omega_{s}}$. 
Definition 2.2 We say that the sequence of spaces $W^{1, p}\left(\Omega_{S}\right)$ is strongly connected with the space $W^{1, p}(\Omega)$ if there exists a sequence of linear continuous operators $l_{S}: W^{1, p}\left(\Omega_{S}\right) \rightarrow$ $W^{1, p}(\Omega)$ such that:

(i) the sequence of norms $\left\|l_{s}\right\|$ is bounded;

(ii) for every $s \in \mathbb{N}$ and every $v \in W^{1, p}\left(\Omega_{s}\right)$, we have $q_{s}\left(l_{s} v\right)=v$ a.e. in $\Omega_{s}$.

Definition 2.3 Let, for every $s \in \mathbb{N}, I_{s}: W^{1, p}\left(\Omega_{s}\right) \rightarrow \mathbb{R}$, and let $I: W^{1, p}(\Omega) \rightarrow \mathbb{R}$. We say that the sequence $\left\{I_{s}\right\} \Gamma$-converges to the functional $I$ if the following conditions are satisfied:

(i) for every function $v \in W^{1, p}(\Omega)$, there exists a sequence $w_{s} \in W^{1, p}\left(\Omega_{s}\right)$ such that $\left\|w_{s}-q_{s} v\right\|_{L^{p}\left(\Omega_{s}\right)} \rightarrow 0$ and $I_{s}\left(w_{s}\right) \rightarrow I(v)$

(ii) for every function $v \in W^{1, p}(\Omega)$ and every sequence $v_{s} \in W^{1, p}\left(\Omega_{S}\right)$ such that $\| v_{S}-$ $q_{s} v \|_{L^{p}\left(\Omega_{s}\right)} \rightarrow 0$, we have $\liminf _{s \rightarrow \infty} I_{S}\left(v_{S}\right) \geqslant I(v)$.

Next, let $c_{1}, c_{2}>0$ and let, for every $s \in \mathbb{N}, \mu_{s} \in L^{1}\left(\Omega_{s}\right)$ and $\mu_{s} \geqslant 0$ in $\Omega_{s}$. We assume that the sequence of norms $\left\|\mu_{s}\right\|_{L^{1}\left(\Omega_{s}\right)}$ is bounded.

Let, for every $s \in \mathbb{N}, f_{s}: \Omega_{s} \times \mathbb{R}^{n} \rightarrow \mathbb{R}$ be a function satisfying the following conditions: for every $\xi \in \mathbb{R}^{n}$, the function $f_{s}(\cdot, \xi)$ is measurable on $\Omega_{s}$; for almost every $x \in \Omega_{s}$, the function $f_{s}(x, \cdot)$ is convex on $\mathbb{R}^{n}$; for almost every $x \in \Omega_{s}$ and every $\xi \in \mathbb{R}^{n}$,

$$
c_{1}|\xi|^{p}-\mu_{s}(x) \leqslant f_{s}(x, \xi) \leqslant c_{2}|\xi|^{p}+\mu_{s}(x) .
$$

In view of the assumptions on the functions $f_{s}$ and $\mu_{s}$, for every $s \in \mathbb{N}$ and every $v \in W^{1, p}\left(\Omega_{s}\right)$, the function $f_{s}(x, \nabla v)$ is summable on $\Omega_{s}$.

Definition 2.4 If $s \in \mathbb{N}$, then $F_{s}: W^{1, p}\left(\Omega_{s}\right) \rightarrow \mathbb{R}$ is the functional such that, for every function $v \in W^{1, p}\left(\Omega_{S}\right)$,

$$
F_{S}(v)=\int_{\Omega_{s}} f_{s}(x, \nabla v) \mathrm{d} x .
$$

By virtue of the conditions on the functions $f_{s}$, for every $s \in \mathbb{N}$, the functional $F_{s}$ is convex and locally bounded. Therefore, for every $s \in \mathbb{N}$, the functional $F_{s}$ is weakly lower semicontinuous.

Next, let $c_{3}>0$ and $c_{4} \geqslant 0$, and let, for every $s \in \mathbb{N}, G_{s}: W^{1, p}\left(\Omega_{s}\right) \rightarrow \mathbb{R}$ be a weakly continuous functional. We assume that, for every $s \in \mathbb{N}$ and every $v \in W^{1, p}\left(\Omega_{s}\right)$,

$$
G_{s}(v) \geqslant c_{3}\|v\|_{L^{p}\left(\Omega_{s}\right)}^{p}-c_{4} .
$$

In view of the weak lower semicontinuity of the functionals $F_{s}$ and the weak continuity of the functionals $G_{s}$, for every $s \in \mathbb{N}$, the functional $F_{s}+G_{s}$ is weakly lower semicontinuous. Moreover, by virtue of inequalities (2.1) and (2.2) and the boundedness of the sequence of norms $\left\|\mu_{s}\right\|_{L^{1}\left(\Omega_{s}\right)}$, there exist positive numbers $c_{5}$ and $c_{6}$ such that, for every $s \in \mathbb{N}$ and every $v \in W^{1, p}\left(\Omega_{s}\right)$, we have

$$
\left(F_{S}+G_{S}\right)(v) \geqslant c_{5}\|v\|_{W^{1, p}\left(\Omega_{s}\right)}^{p}-c_{6} .
$$

For every function $h: \mathbb{R} \rightarrow \mathbb{R}$, we define

$$
\begin{aligned}
& \Phi(h)=\{t \in \mathbb{R}: h(t) \leqslant 0\}, \\
& U(h)=\left\{v \in W^{1, p}(\Omega): h(v) \leqslant 0 \text { a.e. in } \Omega\right\} .
\end{aligned}
$$


Moreover, for every $s \in \mathbb{N}$ and every function $h: \mathbb{R} \rightarrow \mathbb{R}$, we set

$$
U_{s}(h)=\left\{v \in W^{1, p}\left(\Omega_{s}\right): h(v) \leqslant 0 \text { a.e. in } \Omega_{s}\right\} .
$$

It is easy to see that if $h: \mathbb{R} \rightarrow \mathbb{R}$, then the inequalities $\Phi(h) \neq \varnothing$ and $U(h) \neq \varnothing$ are equivalent. Moreover, if $s \in \mathbb{N}, h: \mathbb{R} \rightarrow \mathbb{R}$, and $\Phi(h) \neq \varnothing$, then $U_{s}(h) \neq \varnothing$.

We also note that if $h: \mathbb{R} \rightarrow \mathbb{R}$ and the set $\Phi(h)$ is nonempty and closed, then the set $U(h)$ is sequentially weakly closed in $W^{1, p}(\Omega)$. In fact, let $h: \mathbb{R} \rightarrow \mathbb{R}$ and let the set $\Phi(h)$ has the specified properties. Let $\left\{v_{j}\right\} \subset U(h), v \in W^{1, p}(\Omega)$, and $v_{j} \rightarrow v$ weakly in $W^{1, p}(\Omega)$. Fixing an arbitrary open ball $B \subset \Omega$, we have $\left.\left.v_{j}\right|_{B} \rightarrow v\right|_{B}$ weakly in $W^{1, p}(B)$. Hence, $\left.\left.v_{j}\right|_{B} \rightarrow v\right|_{B}$ strongly in $L^{p}(B)$. This along with the inclusion $\left\{v_{j}\right\} \subset U(h)$ implies that there exist a set $E \subset B$ of measure zero and an increasing sequence $\left\{j_{k}\right\} \subset \mathbb{N}$ such that, for every $x \in B \backslash E, v_{j_{k}}(x) \rightarrow v(x)$ and $\left\{v_{j_{k}}(x)\right\} \subset \Phi(h)$. Then, owing to the closedness of the set $\Phi(h)$, we have $h(v(x)) \leqslant 0$ for every $x \in B \backslash E$. Hence, taking into account the arbitrariness of $B$, we deduce that $h(v) \leqslant 0$ a.e. in $\Omega$. Therefore, $v \in U(h)$ and we conclude that the set $U(h)$ is sequentially weakly closed in $W^{1, p}(\Omega)$.

Similarly, we establish that if $s \in \mathbb{N}, h: \mathbb{R} \rightarrow \mathbb{R}$, and the set $\Phi(h)$ is nonempty and closed, then the set $U_{s}(h)$ is sequentially weakly closed in $W^{1, p}\left(\Omega_{S}\right)$.

Now, in view of the above properties of the functionals $F_{S}+G_{s}$ and due to known results on the existence of minimizers of functionals (see, for instance, [25]), we conclude that if $s \in \mathbb{N}, h: \mathbb{R} \rightarrow \mathbb{R}$, and the set $\Phi(h)$ is nonempty and closed, then there exists a function belonging to the set $U_{s}(h)$ and minimizing the functional $F_{s}+G_{s}$ on this set.

\section{Auxiliary propositions}

We prove a number of auxiliary propositions which will be used in the proof of our main results.

Proposition 3.1 Assume that the embedding of $W^{1, p}(\Omega)$ into $L^{p}(\Omega)$ is compact. Let $v \in$ $W^{1, p}(\Omega)$. Let $\lambda_{1}, \lambda_{2} \in \mathbb{R}$, and let $\lambda_{1}<\lambda_{2}$. Assume that meas $\left\{v \leqslant \lambda_{1}\right\}>0$ and meas $\{v \geqslant$ $\left.\lambda_{2}\right\}>0$. Then meas $\left\{\lambda_{1}<v<\lambda_{2}\right\}>0$.

The proof of this proposition is given in [19]. It uses the Poincaré inequality for elements of the space $W^{1, p}(\Omega)$ which is proved by contradiction essentially taking into account the assumption on the compactness of the embedding of $W^{1, p}(\Omega)$ into $L^{p}(\Omega)$. In this connection, see, for instance, Theorem 1 in [9, Sect. 5.8].

We introduce the following notation: if $\mathcal{E}$ is a domain of $\mathbb{R}^{n}$ and $v: \mathcal{E} \rightarrow \mathbb{R}$, then

$$
\underline{m}(v)=\operatorname{ess} \inf _{\mathcal{E}} v, \quad \bar{m}(v)=\operatorname{ess} \sup _{\mathcal{E}} v .
$$

Proposition 3.2 Assume that the embedding of $W^{1, p}(\Omega)$ into $L^{p}(\Omega)$ is compact. Let $h$ : $\mathbb{R} \rightarrow \mathbb{R}$ be a function such that the set $\Phi(h)$ is nonempty and closed. Let $v \in U(h)$. Then the following assertions hold:

(a) if $\underline{m}(v)<\bar{m}(v)$, then $(\underline{m}(v), \bar{m}(v)) \subset \Phi(h)$;

(b) if $\underline{m}(v) \in \mathbb{R}$, then $\underline{m}(v) \in \Phi(h)$;

(c) if $\overline{\bar{m}}(v) \in \mathbb{R}$, then $\overline{\bar{m}}(v) \in \Phi(h)$;

(d) if $\underline{m}(v), \bar{m}(v) \in \mathbb{R}$, then $[\underline{m}(v), \bar{m}(v)] \subset \Phi(h)$.

The proof of this result is based on the use of Proposition 3.1. For details of the proof, see Proposition 2 in [19].

Proposition 3.2 allows us to conclude that the following result holds. 
Proposition 3.3 Let $s \in \mathbb{N}$, and let the embedding of $W^{1, p}\left(\Omega_{s}\right)$ into $L^{p}\left(\Omega_{s}\right)$ be compact. Let $h: \mathbb{R} \rightarrow \mathbb{R}$ be a function such that the set $\Phi(h)$ is nonempty and closed. Let $v \in U_{S}(h)$. Then the following assertions hold:

(a) if $\underline{m}(v)<\bar{m}(v)$, then $(\underline{m}(v), \bar{m}(v)) \subset \Phi(h)$;

(b) if $\underline{m}(v) \in \mathbb{R}$, then $\underline{m}(v) \in \Phi(h)$;

(c) if $\bar{m}(v) \in \mathbb{R}$, then $\bar{m}(v) \in \Phi(h)$;

(d) if $\underline{m}(v), \bar{m}(v) \in \mathbb{R}$, then $[\underline{m}(v), \bar{m}(v)] \subset \Phi(h)$.

Remark 3.4 In connection with the latter proposition and the next result, we note that if the embedding of $W^{1, p}(\Omega)$ into $L^{p}(\Omega)$ is compact and the sequence of spaces $W^{1, p}\left(\Omega_{S}\right)$ is strongly connected with the space $W^{1, p}(\Omega)$, then, for every $s \in \mathbb{N}$, the embedding of $W^{1, p}\left(\Omega_{s}\right)$ into $L^{p}\left(\Omega_{s}\right)$ is compact.

Proposition 3.5 Assume that the embedding of $W^{1, p}(\Omega)$ into $L^{p}(\Omega)$ is compact and the sequence of spaces $W^{1, p}\left(\Omega_{s}\right)$ is strongly connected with the space $W^{1, p}(\Omega)$. Let, for every $s \in \mathbb{N}, h_{s}: \mathbb{R} \rightarrow \mathbb{R}$ be a function such that the set $\Phi\left(h_{s}\right)$ is nonempty and closed. Let $h: \mathbb{R} \rightarrow \mathbb{R}$ be a function such that the set $\Phi(h)$ is nonempty. Assume that the following condition is satisfied:

(*) if $t_{s} \rightarrow t$ in $\mathbb{R},\left\{\tilde{s}_{j}\right\}$ is an increasing sequence in $\mathbb{N}$, and, for every $j \in \mathbb{N}$, we have $t_{\tilde{s}_{j}} \in \Phi\left(h_{\tilde{s}_{j}}\right)$, then $t \in \Phi(h)$.

Let, for every $s \in \mathbb{N}, v_{s} \in U_{s}\left(h_{s}\right)$. Assume that the sequence of norms $\left\|v_{s}\right\|_{W^{1, p}\left(\Omega_{s}\right)}$ is bounded.

Then there exist an increasing sequence $\left\{s_{j}\right\} \subset \mathbb{N}$ and a function $v \in U(h)$ such that $\left\|v_{s_{j}}-q_{s_{j}} v\right\|_{L^{p}\left(\Omega_{s_{j}}\right)} \rightarrow 0$.

Proof Since the sequence of spaces $W^{1, p}\left(\Omega_{s}\right)$ is strongly connected with the space $W^{1, p}(\Omega)$, there exists a sequence of linear continuous operators $l_{s}: W^{1, p}\left(\Omega_{s}\right) \rightarrow W^{1, p}(\Omega)$ such that the sequence of norms $\left\|l_{s}\right\|$ is bounded and

$$
\forall s \in \mathbb{N}, \quad q_{s}\left(l_{s} v_{s}\right)=v_{s} \text { a.e. in } \Omega_{s} .
$$

The boundedness of the sequences of norms $\left\|l_{S}\right\|$ and $\left\|v_{S}\right\|_{W^{1, p}\left(\Omega_{s}\right)}$ implies that the sequence $\left\{l_{s} v_{s}\right\}$ is bounded in $W^{1, p}(\Omega)$.

We denote by $\alpha$ the lower limit of the sequence $\left\{\right.$ meas $\Omega_{s}$. There are two logical possibilities: either $\alpha=0$ or $\alpha>0$.

First, we consider the case $\alpha=0$. In this case, there exists an increasing sequence $\left\{\bar{s}_{k}\right\} \subset \mathbb{N}$ such that

$$
\text { meas } \Omega_{\bar{s}_{k}} \rightarrow 0 \text {. }
$$

Taking into account the reflexivity of the space $W^{1, p}(\Omega)$ and the compactness of the embedding of $W^{1, p}(\Omega)$ into $L^{p}(\Omega)$, we deduce from the boundedness of the sequence $\left\{l_{s} v_{s}\right\}$ in $W^{1, p}(\Omega)$ that there exist an increasing sequence $\left\{s_{j}\right\} \subset\left\{\bar{s}_{k}\right\}$ and a function $w \in W^{1, p}(\Omega)$ such that $l_{s_{j}} v_{s_{j}} \rightarrow w$ strongly in $L^{p}(\Omega)$. Then, in view of (3.1), we have

$$
\left\|v_{s_{j}}-q_{s_{j}} w\right\|_{L^{p}\left(\Omega_{s_{j}}\right)} \rightarrow 0 .
$$

Since the set $\Phi(h)$ is nonempty, there exists $t_{*} \in \Phi(h)$. Let $v: \Omega \rightarrow \mathbb{R}$ be the function such that, for every $x \in \Omega, v(x)=t_{*}$. Obviously, $v \in U(h)$. By virtue of (3.2), we have $\left\|q_{s_{j}}(w-v)\right\|_{L^{p}\left(\Omega_{s_{j}}\right)} \rightarrow 0$. This and (3.3) imply that $\left\|v_{s_{j}}-q_{s_{j}} v\right\|_{L^{p}\left(\Omega_{s_{j}}\right)} \rightarrow 0$. Thus, in the case $\alpha=0$, the conclusion of the proposition holds. 
Now, we consider the case $\alpha>0$. In this case, the following inequalities hold:

$$
\begin{aligned}
& \liminf _{s \rightarrow \infty} \bar{m}\left(v_{s}\right)>-\infty, \\
& \limsup _{s \rightarrow \infty} \underline{m}\left(v_{s}\right)<+\infty .
\end{aligned}
$$

In fact, let $C$ be a majorant of the sequence of norms $\left\|v_{S}\right\|_{W^{1, p}\left(\Omega_{s}\right)}$, and let $M>C(2 / \alpha)^{1 / p}$. Suppose that inequality (3.4) does not hold. Then there exists $r \in \mathbb{N}$ such that

$$
\bar{m}\left(v_{r}\right)<-M, \quad \text { meas } \Omega_{r} \geqslant \alpha / 2 .
$$

The first inequality in (3.6) implies that $\left|v_{r}\right| \geqslant M$ a.e. in $\Omega_{r}$. Then, using the second inequality in (3.6), we obtain

$$
\left\|v_{r}\right\|_{W^{1, p}\left(\Omega_{r}\right)}^{p} \geqslant \int_{\Omega_{r}}\left|v_{r}\right|^{p} \mathrm{~d} x \geqslant M^{p} \text { meas } \Omega_{r} \geqslant M^{p} \alpha / 2>C^{p} .
$$

Hence, $\left\|v_{r}\right\|_{W^{1, p}\left(\Omega_{r}\right)}>C$. However, this contradicts the fact that $C$ is a majorant of the sequence of norms $\left\|v_{s}\right\|_{W^{1, p}\left(\Omega_{S}\right)}$. The obtained contradiction proves that inequality (3.4) holds. Similarly, we establish that inequality (3.5) also holds.

Further, we assume that

$$
\begin{gathered}
\limsup _{s \rightarrow \infty} \bar{m}\left(v_{s}\right)<+\infty, \\
\liminf _{s \rightarrow \infty} \underline{m}\left(v_{s}\right)>-\infty .
\end{gathered}
$$

In view of these inequalities, there exist $M_{1}, M_{2} \in \mathbb{R}$ and $\hat{s} \in \mathbb{N}$ such that, for every $s \in \mathbb{N}$, $s>\hat{s}$,

$$
M_{1} \leqslant \underline{m}\left(v_{s}\right) \leqslant \bar{m}\left(v_{s}\right) \leqslant M_{2} .
$$

Taking into account Remark 3.4 and inequality (3.9), we derive from Proposition 3.3 that, for every $s \in \mathbb{N}, s>\hat{s}$,

$$
\left[\underline{m}\left(v_{s}\right), \bar{m}\left(v_{s}\right)\right] \subset \Phi\left(h_{s}\right) .
$$

For every $s \in \mathbb{N}, s \leqslant \hat{s}$, we set $w_{s}=l_{s} v_{s}$. For every $s \in \mathbb{N}, s>\hat{s}$, we define

$$
w_{s}=\min \left\{\max \left\{l_{s} v_{s}, \underline{m}\left(v_{s}\right)\right\}, \bar{m}\left(v_{s}\right)\right\} .
$$

Obviously, $\left\{w_{s}\right\} \subset W^{1, p}(\Omega)$. Moreover, in view of (3.1), for every $s \in \mathbb{N}, s>\hat{s}$,

$$
q_{s} w_{s}=v_{s} \text { a.e. in } \Omega_{s} .
$$

The boundedness of the sequence $\left\{l_{s} v_{s}\right\}$ in $W^{1, p}(\Omega)$ along with inequality (3.9) implies that the sequence $\left\{w_{s}\right\}$ is bounded in $W^{1, p}(\Omega)$. Then, owing to the reflexivity of the space $W^{1, p}(\Omega)$ and the compactness of the embedding of $W^{1, p}(\Omega)$ into $L^{p}(\Omega)$, there exist an increasing sequence $\left\{s_{j}\right\} \subset \mathbb{N}$ and a function $v \in W^{1, p}(\Omega)$ such that $\left\{s_{j}\right\} \subset(\hat{s},+\infty)$,

$$
w_{s_{j}} \rightarrow v \text { strongly in } L^{p}(\Omega),
$$

and $w_{s_{j}} \rightarrow v$ a.e. in $\Omega$. According to the latter property, there exists a set $E \subset \Omega$ of measure zero such that

$$
\forall x \in \Omega \backslash E, \quad w_{s_{j}}(x) \rightarrow v(x) .
$$

We fix an arbitrary $x \in \Omega \backslash E$, and let $\left\{t_{s}\right\}$ be the sequence of numbers such that: $t_{s}=w_{s}(x)$ if $s=s_{j}$ for some $j \in \mathbb{N} ; t_{s}=v(x)$ if $s \neq s_{j}$ for every $j \in \mathbb{N}$. Due to (3.13), we have $t_{s} \rightarrow v(x)$. Moreover, taking into account the definition of the functions $w_{s}$ and using the inclusion 
$\left\{s_{j}\right\} \subset(\hat{s},+\infty)$ along with (3.10), we find that, for every $j \in \mathbb{N}, t_{s_{j}} \in \Phi\left(h_{s_{j}}\right)$. Then, by virtue of condition (*), we obtain the inclusion $v(x) \in \Phi(h)$. Hence, $h(v(x)) \leqslant 0$. Therefore, $v \in U(h)$. In addition, it follows from (3.11) and (3.12) that $\left\|v_{s_{j}}-q_{s_{j}} v\right\|_{L^{p}\left(\Omega_{s_{j}}\right)} \rightarrow 0$. Thus, if inequalities (3.7) and (3.8) are satisfied, then the conclusion of the proposition holds.

Now, we assume that inequality (3.7) is satisfied and inequality (3.8) is not satisfied. The assumption that inequality (3.8) is not satisfied implies that, for an increasing sequence $\left\{i_{k}\right\} \subset \mathbb{N}$,

$$
\underline{m}\left(v_{i_{k}}\right) \rightarrow-\infty \text {. }
$$

In view of (3.4), (3.7), and (3.14), there exist $M_{3}>0$ and $k^{\prime} \in \mathbb{N}$ such that, for every $k \in \mathbb{N}$, $k>k^{\prime}$,

$$
\begin{aligned}
\left|\bar{m}\left(v_{i_{k}}\right)\right| & \leqslant M_{3}, \\
\underline{m}\left(v_{i_{k}}\right) & <-M_{3} .
\end{aligned}
$$

We define the sequence $\left\{z_{k}\right\}$ as follows: if $k \in \mathbb{N}, k \leqslant k^{\prime}$, then $z_{k}=l_{i_{k}} v_{i_{k}}$; if $k \in \mathbb{N}, k>k^{\prime}$, then $z_{k}=\min \left\{l_{i_{k}} v_{i_{k}}, \bar{m}\left(v_{i_{k}}\right)\right\}$. Obviously, $\left\{z_{k}\right\} \subset W^{1, p}(\Omega)$. In addition, owing to (3.15) and the boundedness of the sequence $\left\{l_{s} v_{s}\right\}$ in $W^{1, p}(\Omega)$, the sequence $\left\{z_{k}\right\}$ is bounded in $W^{1, p}(\Omega)$. Then, due to the reflexivity of the space $W^{1, p}(\Omega)$ and the compactness of the embedding of $W^{1, p}(\Omega)$ into $L^{p}(\Omega)$, there exist an increasing sequence $\left\{k_{j}\right\} \subset \mathbb{N}$ and a function $v \in W^{1, p}(\Omega)$ such that $\left\{k_{j}\right\} \subset\left(k^{\prime},+\infty\right)$,

$$
\begin{aligned}
& z_{k_{j}} \rightarrow v \text { strongly in } L^{p}(\Omega), \\
& z_{k_{j}} \rightarrow v \text { a.e. in } \Omega .
\end{aligned}
$$

For every $j \in \mathbb{N}$, we set $s_{j}=i_{k_{j}}$. Since $\left\{k_{j}\right\} \subset\left(k^{\prime},+\infty\right)$, it follows from (3.15) and (3.16) that, for every $j \in \mathbb{N}, \underline{m}\left(v_{s_{j}}\right)<\bar{m}\left(v_{s_{j}}\right)$ and $\bar{m}\left(v_{s_{j}}\right) \in \mathbb{R}$. Then, taking into account Remark 3.4 and applying Proposition 3.3, we find that

$$
\forall j \in \mathbb{N}, \quad\left(\underline{m}\left(v_{s_{j}}\right), \bar{m}\left(v_{s_{j}}\right)\right] \subset \Phi\left(h_{s_{j}}\right) .
$$

In view of (3.18), there exists a set $E \subset \Omega$ of measure zero such that

$$
\forall x \in \Omega \backslash E, \quad z_{k_{j}}(x) \rightarrow v(x) .
$$

We fix $x \in \Omega \backslash E$. Owing to (3.14) and (3.20) and in view of the definition of the functions $z_{k}$, there exists $j_{1} \in \mathbb{N}$ such that, for every $j \in \mathbb{N}, j \geqslant j_{1}$, we have $\underline{m}\left(v_{s_{j}}\right)<z_{k_{j}}(x) \leqslant \bar{m}\left(v_{s_{j}}\right)$. This and (3.19) imply that, for every $j \in \mathbb{N}, j \geqslant j_{1}$,

$$
z_{k_{j}}(x) \in \Phi\left(h_{s_{j}}\right) .
$$

We define the sequence $\left\{t_{s}\right\}$ as follows: $t_{s}=z_{k_{j+j_{1}}}(x)$ if $s=s_{j+j_{1}}$ for some $j \in \mathbb{N} ; t_{s}=v(x)$ if $s \neq s_{j+j_{1}}$ for every $j \in \mathbb{N}$. In addition, for every $j \in \mathbb{N}$, we set $\tilde{s}_{j}=s_{j+j_{1}}$. By virtue of (3.20), we have $t_{s} \rightarrow v(x)$. Moreover, in view of (3.21), for every $j \in \mathbb{N}$, we have $t_{\tilde{s}_{j}} \in \Phi\left(h_{\tilde{s}_{j}}\right)$. Then, using condition $(*)$, we find that $v(x) \in \Phi(h)$. Hence, $h(v(x)) \leqslant 0$. Therefore, $v \in U(h)$. In addition, taking into account the definition of the functions $z_{k}$ and using (3.1) and (3.17), we establish that $\left\|v_{s_{j}}-q_{s_{j}} v\right\|_{L^{p}\left(\Omega_{s_{j}}\right)} \rightarrow 0$. Thus, if inequality (3.7) is satisfied and inequality (3.8) is not satisfied, then the conclusion of the proposition holds.

As an intermediate result, we conclude that if inequality (3.7) is satisfied, then the conclusion of the proposition holds. 
Now, we assume that inequality (3.7) is not satisfied. Then there exist an increasing sequence $\left\{i_{k}\right\} \subset \mathbb{N}$ and an element $\beta \in \overline{\mathbb{R}}$ such that

$$
\begin{aligned}
& \bar{m}\left(v_{i_{k}}\right) \rightarrow+\infty, \\
& \underline{m}\left(v_{i_{k}}\right) \rightarrow \beta .
\end{aligned}
$$

It follows from (3.5) and (3.23) that $\beta<+\infty$.

Suppose that $\beta \in \mathbb{R}$. Then, owing to (3.22) and (3.23), there exists $k^{\prime \prime} \in \mathbb{N}$ such that, for every $k \in \mathbb{N}, k>k^{\prime \prime}$,

$$
\begin{aligned}
\bar{m}\left(v_{i_{k}}\right) & >|\beta|+1, \\
\left|\underline{m}\left(v_{i_{k}}\right)\right| & \leqslant|\beta|+1 .
\end{aligned}
$$

We define the sequence $\left\{y_{k}\right\}$ as follows: if $k \in \mathbb{N}, k \leqslant k^{\prime \prime}$, then $y_{k}=l_{i_{k}} v_{i_{k}}$; if $k \in \mathbb{N}, k>k^{\prime \prime}$, then $y_{k}=\max \left\{l_{i_{k}} v_{i_{k}}, \underline{m}\left(v_{i_{k}}\right)\right\}$. Obviously, $\left\{y_{k}\right\} \subset W^{1, p}(\Omega)$. In addition, owing to (3.25) and the boundedness of the sequence $\left\{l_{s} v_{s}\right\}$ in $W^{1, p}(\Omega)$, the sequence $\left\{y_{k}\right\}$ is bounded in $W^{1, p}(\Omega)$. Then, due to the reflexivity of the space $W^{1, p}(\Omega)$ and the compactness of the embedding of $W^{1, p}(\Omega)$ into $L^{p}(\Omega)$, there exist an increasing sequence $\left\{k_{j}\right\} \subset \mathbb{N}$ and a function $v \in W^{1, p}(\Omega)$ such that $\left\{k_{j}\right\} \subset\left(k^{\prime \prime},+\infty\right)$,

$$
\begin{aligned}
& y_{k_{j}} \rightarrow v \text { strongly in } L^{p}(\Omega), \\
& y_{k_{j}} \rightarrow v \text { a.e. in } \Omega .
\end{aligned}
$$

For every $j \in \mathbb{N}$, we set $s_{j}=i_{k_{j}}$. Since $\left\{k_{j}\right\} \subset\left(k^{\prime \prime},+\infty\right)$, it follows from (3.24) and (3.25) that, for every $j \in \mathbb{N}, \underline{m}\left(v_{s_{j}}\right)<\bar{m}\left(v_{s_{j}}\right)$ and $\underline{m}\left(v_{s_{j}}\right) \in \mathbb{R}$. Then, taking into account Remark 3.4 and applying Proposition 3.3, we find that

$$
\forall j \in \mathbb{N}, \quad\left[\underline{m}\left(v_{s_{j}}\right), \bar{m}\left(v_{s_{j}}\right)\right) \subset \Phi\left(h_{s_{j}}\right) .
$$

In view of (3.27), there exists a set $E \subset \Omega$ of measure zero such that

$$
\forall x \in \Omega \backslash E, \quad y_{k_{j}}(x) \rightarrow v(x) .
$$

We fix $x \in \Omega \backslash E$. Owing to (3.22) and (3.29) and in view of the definition of the functions $y_{k}$, there exists $j_{1} \in \mathbb{N}$ such that, for every $j \in \mathbb{N}, j \geqslant j_{1}$, we have $\underline{m}\left(v_{s_{j}}\right) \leqslant y_{k_{j}}(x)<\bar{m}\left(v_{s_{j}}\right)$. This and (3.28) imply that, for every $j \in \mathbb{N}, j \geqslant j_{1}$,

$$
y_{k_{j}}(x) \in \Phi\left(h_{s_{j}}\right) .
$$

We define the sequence $\left\{t_{s}\right\}$ as follows: $t_{s}=y_{k_{j+j_{1}}}(x)$ if $s=s_{j+j_{1}}$ for some $j \in \mathbb{N} ; t_{s}=v(x)$ if $s \neq s_{j+j_{1}}$ for every $j \in \mathbb{N}$. In addition, for every $j \in \mathbb{N}$, we set $\tilde{s}_{j}=s_{j+j_{1}}$. By virtue of (3.29), we have $t_{s} \rightarrow v(x)$. Moreover, in view of (3.30), for every $j \in \mathbb{N}$, we have $t_{\tilde{s}_{j}} \in \Phi\left(h_{\tilde{s}_{j}}\right)$. Then, using condition $(*)$, we find that $v(x) \in \Phi(h)$. Hence, $h(v(x)) \leqslant 0$. Therefore, $v \in U(h)$. In addition, taking into account the definition of the functions $y_{k}$ and using (3.1) and (3.26), we establish that $\left\|v_{s_{j}}-q_{s_{j}} v\right\|_{L^{p}\left(\Omega_{s_{j}}\right)} \rightarrow 0$. Thus, if $\beta \in \mathbb{R}$, then the conclusion of the proposition holds.

Let $\beta=-\infty$. Then, by virtue of (3.22) and (3.23) and due to the reflexivity of the space $W^{1, p}(\Omega)$ and the compactness of the embedding of $W^{1, p}(\Omega)$ into $L^{p}(\Omega)$, there exist an increasing sequence $\left\{s_{j}\right\} \subset \mathbb{N}$ and a function $v \in W^{1, p}(\Omega)$ such that

$$
\begin{gathered}
\underline{m}\left(v_{s_{j}}\right) \rightarrow-\infty, \quad \bar{m}\left(v_{s_{j}}\right) \rightarrow+\infty, \\
l_{s_{j}} v_{s_{j}} \rightarrow v \text { strongly in } L^{p}(\Omega) .
\end{gathered}
$$


We fix an arbitrary $x \in \Omega$. In view of (3.31), there exists $j_{1} \in \mathbb{N}$ such that, for every $j \in \mathbb{N}$, $j \geqslant j_{1}$, we have $\underline{m}\left(v_{s_{j}}\right)<v(x)<\bar{m}\left(v_{s_{j}}\right)$. Therefore, taking into account Remark 3.4 and applying Proposition 3.3, we find that if $j \in \mathbb{N}, j \geqslant j_{1}$, then $v(x) \in \Phi\left(h_{s_{j}}\right)$. For every $s \in \mathbb{N}$, we set $t_{s}=v(x)$. Moreover, for every $j \in \mathbb{N}$, we define $\tilde{s}_{j}=s_{j+j_{1}}$. Obviously, $t_{s} \rightarrow v(x)$ in $\mathbb{R},\left\{\tilde{s}_{j}\right\}$ is an increasing sequence in $\mathbb{N}$, and, for every $j \in \mathbb{N}, t_{\tilde{s}_{j}} \in \Phi\left(h_{\tilde{s}_{j}}\right)$. Then, by virtue of condition (*), we have $v(x) \in \Phi(h)$. Hence, $h(v(x)) \leqslant 0$. Therefore, $v \in U(h)$. In addition, it follows from (3.1) and (3.32) that $\left\|v_{s_{j}}-q_{s_{j}} v\right\|_{L^{p}\left(\Omega_{s_{j}}\right)} \rightarrow 0$. Thus, if $\beta=-\infty$, then the conclusion of the proposition holds.

Consequently, if inequality (3.7) is not satisfied, then the conclusion of the proposition holds. Thus, in the case $\alpha>0$, the conclusion of the proposition holds.

Proposition 3.6 Assume that the embedding of $W^{1, p}(\Omega)$ into $L^{p}(\Omega)$ is compact. Let, for every $s \in \mathbb{N}, h_{s}: \mathbb{R} \rightarrow \mathbb{R}$. Let $h: \mathbb{R} \rightarrow \mathbb{R}$ be a function such that the set $\Phi(h)$ is closed and has nonempty interior. Assume that the following condition is satisfied:

$(\bar{*})$ if $t_{1}, t_{2} \in \mathbb{R}, t_{1}<t_{2},\left(t_{1}, t_{2}\right) \subset \Phi(h)$, and $0<\sigma<\left(t_{2}-t_{1}\right) / 2$, then there exists $\bar{s} \in \mathbb{N}$ such that, for every $s \in \mathbb{N}, s \geqslant \bar{s}$, we have $\left[t_{1}+\sigma, t_{2}-\sigma\right] \subset \Phi\left(h_{s}\right)$.

Let $v \in U(h)$, and let $\underline{m}(v)<\bar{m}(v)$. Let, for every $s \in \mathbb{N}, v_{s} \in W^{1, p}\left(\Omega_{s}\right)$. Assume that

$$
\left\|v_{s}-q_{s} v\right\|_{L^{p}\left(\Omega_{s}\right)} \rightarrow 0 .
$$

Then there exist a sequence of functions $w_{s} \in W^{1, p}\left(\Omega_{s}\right)$, sequences of measurable sets $E_{s} \subset \Omega_{s}$ and $\tilde{E}_{s} \subset \Omega_{s}$, a sequence $\left\{\beta_{s}\right\} \subset(0,1]$, and a number $\tilde{s} \in \mathbb{N}$ such that the following assertions hold:

(a) $\left\|w_{s}-q_{s} v\right\|_{L^{p}\left(\Omega_{s}\right)} \rightarrow 0$;

(b) for every $s \in \mathbb{N}, \nabla w_{s}=\beta_{s}\left(\nabla v_{s} \cdot 1_{\Omega_{s} \backslash E_{s}}+\nabla\left(q_{s} v\right) \cdot 1_{E_{s} \backslash \tilde{E}_{s}}\right)$ a.e. in $\Omega_{s}$;

(c) for every $s \in \mathbb{N}$, $s \geqslant \tilde{s}$, we have $w_{s} \in U_{s}\left(h_{s}\right)$;

(d) meas $E_{s} \rightarrow 0$;

(e) for every $s \in \mathbb{N}, \tilde{E}_{s} \subset E_{s}$;

(f) $\beta_{s} \rightarrow 1$.

Proof The following cases are possible:

(i) $\underline{m}(v), \bar{m}(v) \in \mathbb{R}$;

(ii) $\underline{m}(v) \in \mathbb{R}, \bar{m}(v)=+\infty$;

(iii) $\underline{m}(v)=-\infty, \bar{m}(v) \in \mathbb{R}$;

(iv) $\underline{m}(v)=-\infty, \bar{m}(v)=+\infty$.

We will prove the validity of the conclusion of the proposition in each of these cases.

Let us consider case (i). By virtue of Proposition 3.2, we have

$$
[\underline{m}(v), \bar{m}(v)] \subset \Phi(h),
$$

and, in view of the definition of $\underline{m}(v)$ and $\bar{m}(v)$, there exists a set $E \subset \Omega$ of measure zero such that

$$
\forall x \in \Omega \backslash E, \quad \underline{m}(v) \leqslant v(x) \leqslant \bar{m}(v) .
$$

We define

$$
\alpha=\frac{1}{2}(\bar{m}(v)-\underline{m}(v))
$$

and fix a sequence $\left\{\sigma_{k}\right\} \subset(0, \alpha)$ such that

$$
\sigma_{k} \rightarrow 0 .
$$


For every $k \in \mathbb{N}$, we set

$$
\alpha_{k}=\frac{\alpha}{\alpha+2 \sigma_{k}}
$$

Obviously, $\left\{\alpha_{k}\right\} \subset(0,1)$ and

$$
\alpha_{k} \rightarrow 1
$$

For every $k, s \in \mathbb{N}$, we set

$$
\begin{aligned}
v_{s}^{(k)} & =\min \left\{\max \left\{v_{s}, q_{s} v-\sigma_{k}\right\}, q_{s} v+\sigma_{k}\right\}, \\
E_{s}^{(k)} & =\left\{\left|v_{s}-q_{s} v\right| \geqslant \sigma_{k}\right\} .
\end{aligned}
$$

If $k, s \in \mathbb{N}$, then we have

$$
\begin{aligned}
v_{s}^{(k)} & \in W^{1, p}\left(\Omega_{s}\right), \\
q_{s} v-\sigma_{k} & \leqslant v_{s}^{(k)} \leqslant q_{s} v+\sigma_{k} \text { in } \Omega_{s}, \\
\left\|v_{s}^{(k)}-q_{s} v\right\|_{L^{p}\left(\Omega_{s}\right)} & \leqslant \sigma_{k}(\text { meas } \Omega)^{1 / p}, \\
\nabla v_{s}^{(k)} & =\nabla v_{s} \cdot 1_{\Omega_{s} \backslash E_{s}^{(k)}}+\nabla\left(q_{s} v\right) \cdot 1_{E_{s}^{(k)}} \text { a.e. in } \Omega_{s}, \\
\text { meas } E_{s}^{(k)} & \leqslant \sigma_{k}^{-p}\left\|v_{s}-q_{s} v\right\|_{L^{p}\left(\Omega_{s}\right)}^{p} .
\end{aligned}
$$

Next, for every $k, s \in \mathbb{N}$, we set

$$
w_{s}^{(k)}=\alpha_{k} v_{s}^{(k)}+\left(1-\alpha_{k}\right) \underline{m}(v)+2 \alpha_{k} \sigma_{k} .
$$

Using (3.35), (3.36), (3.38), and (3.40)-(3.43), we find that, for every $k, s \in \mathbb{N}$,

$$
\begin{aligned}
w_{s}^{(k)} \in & W^{1, p}\left(\Omega_{s}\right), \\
x \in & \Omega_{s} \backslash E \Longrightarrow w_{s}^{(k)}(x) \in\left[\underline{m}(v)+\alpha_{k} \sigma_{k}, \bar{m}(v)-\alpha_{k} \sigma_{k}\right], \\
\left\|w_{s}^{(k)}-q_{s} v\right\|_{L^{p}\left(\Omega_{s}\right) \leqslant} & 3 \sigma_{k}(\operatorname{meas} \Omega)^{1 / p} \\
& +\left(1-\alpha_{k}\right)\left\{|\underline{m}(v)|(\operatorname{meas} \Omega)^{1 / p}+\|v\|_{L^{p}(\Omega)}\right\}, \\
\nabla w_{s}^{(k)}= & \alpha_{k}\left(\nabla v_{s} \cdot 1_{\Omega_{s} \backslash E_{s}^{(k)}}+\nabla\left(q_{s} v\right) \cdot 1_{E_{s}^{(k)}}\right) \text { a.e. in } \Omega_{s} .
\end{aligned}
$$

In addition to the above-defined functions and sets, we introduce an increasing sequence $\left\{s_{k}\right\} \subset \mathbb{N}$. First of all, in view of inclusion (3.34), the inclusion $\left\{\alpha_{k} \sigma_{k}\right\} \subset(0, \alpha)$, and condition ( $\bar{*})$, there exists an increasing sequence $\left\{s_{k}^{\prime}\right\} \subset \mathbb{N}$ such that, for every $k \in \mathbb{N}$ and every $s \in \mathbb{N}, s \geqslant s_{k}^{\prime}$,

$$
\left[\underline{m}(v)+\alpha_{k} \sigma_{k}, \bar{m}(v)-\alpha_{k} \sigma_{k}\right] \subset \Phi\left(h_{s}\right) .
$$

Moreover, by virtue of (3.33) and (3.44), there exists an increasing sequence $\left\{s_{k}^{\prime \prime}\right\} \subset \mathbb{N}$ such that, for every $k \in \mathbb{N}$ and every $s \in \mathbb{N}, s \geqslant s_{k}^{\prime \prime}$,

$$
\text { meas } E_{s}^{(k)} \leqslant \sigma_{k} \text {. }
$$

For every $k \in \mathbb{N}$, we set $s_{k}=\max \left\{s_{k}^{\prime}, s_{k}^{\prime \prime}\right\}$. Obviously, the sequence $\left\{s_{k}\right\}$ is increasing.

We define the sequence of functions $w_{s}$ as follows:

$$
w_{s}=\left\{\begin{array}{l}
w_{s}^{(1)} \text { if } s \leqslant s_{1}, \\
w_{s}^{(k)} \text { if } s_{k}<s \leqslant s_{k+1}, k \in \mathbb{N} .
\end{array}\right.
$$

Moreover, let $\left\{E_{s}\right\}$ be the sequence of sets such that

$$
E_{s}=\left\{\begin{array}{l}
E_{s}^{(1)} \text { if } s \leqslant s_{1} \\
E_{s}^{(k)} \text { if } s_{k}<s \leqslant s_{k+1}, k \in \mathbb{N} .
\end{array}\right.
$$


For every $s \in \mathbb{N}$, we define $\tilde{E}_{s}=\varnothing$. In addition, let $\left\{\beta_{s}\right\}$ be the sequence of numbers such that

$$
\beta_{s}=\left\{\begin{array}{l}
\alpha_{1} \text { if } s \leqslant s_{1} \\
\alpha_{k} \text { if } s_{k}<s \leqslant s_{k+1}, k \in \mathbb{N} .
\end{array}\right.
$$

Finally, we set $\tilde{s}=s_{1}+1$.

Owing to (3.45), for every $s \in \mathbb{N}$, we have $w_{s} \in W^{1, p}\left(\Omega_{s}\right)$. Moreover, for every $s \in \mathbb{N}$, the sets $E_{s}$ and $\tilde{E}_{s}$ are contained in $\Omega_{s}$ and are measurable. It is also clear that $\left\{\beta_{s}\right\} \subset(0,1]$ and $\tilde{s} \in \mathbb{N}$. Using (3.37), (3.39), and (3.47), we establish that assertion (a) holds. In view of (3.48), assertion (b) holds. From (3.46) and (3.49), we derive that assertion (c) holds. Using (3.37) and (3.50), we find that assertion (d) holds. Assertion (e) is obvious. Finally, because of (3.39), assertion (f) holds. Thus, in case (i), the conclusion of the proposition holds.

Now, we consider case (ii). By virtue of Proposition 3.2, we have

$$
[\underline{m}(v),+\infty) \subset \Phi(h),
$$

and, in view of the definition of $\underline{m}(v)$, there exists a set $E \subset \Omega$ of measure zero such that

$$
\forall x \in \Omega \backslash E, \quad v(x) \geqslant \underline{m}(v) .
$$

We fix a sequence $\left\{\sigma_{k}\right\} \subset(0,1)$ with the property (3.37).

For every $k, s \in \mathbb{N}$, we set

$$
v_{s}^{(k)}=\max \left\{v_{s}, q_{s} v-\sigma_{k}\right\}+2 \sigma_{k} .
$$

If $k, s \in \mathbb{N}$, then we have $v_{s}^{(k)} \in W^{1, p}\left(\Omega_{s}\right)$,

$$
\begin{aligned}
v_{s}^{(k)} & \geqslant q_{s} v+\sigma_{k} \text { in } \Omega_{s}, \\
\left\|v_{s}^{(k)}-q_{s} v\right\|_{L^{p}\left(\Omega_{s}\right)} & \leqslant\left\|v_{s}-q_{s} v\right\|_{L^{p}\left(\Omega_{s}\right)}+2 \sigma_{k}(\text { meas } \Omega)^{1 / p} .
\end{aligned}
$$

Next, for every $k, s \in \mathbb{N}$, we set

$$
\begin{aligned}
& w_{s}^{(k)}=\min \left\{v_{s}^{(k)},|\underline{m}(v)|+k\right\}, \\
& E_{s}^{(k)}=\left\{v_{s} \leqslant q_{s} v-\sigma_{k}\right\} \cup\left\{v_{s}^{(k)} \geqslant|\underline{m}(v)|+k\right\}, \\
& \tilde{E}_{s}^{(k)}=\left\{v_{s}^{(k)} \geqslant|\underline{m}(v)|+k\right\} .
\end{aligned}
$$

It is clear that if $k, s \in \mathbb{N}$, then $w_{s}^{(k)} \in W^{1, p}\left(\Omega_{s}\right)$ and

$$
\nabla w_{s}^{(k)}=\nabla v_{s} \cdot 1_{\Omega_{s} \backslash E_{s}^{(k)}}+\nabla\left(q_{s} v\right) \cdot 1_{E_{s}^{(k)} \backslash \tilde{E}_{s}^{(k)}} \text { a.e. in } \Omega_{s} .
$$

Moreover, using (3.52)-(3.54) and the definitions of the functions $w_{s}^{(k)}$ and the sets $E_{s}^{(k)}$ and $\tilde{E}_{s}^{(k)}$, we find that, for every $k, s \in \mathbb{N}$,

$$
\begin{aligned}
x \in \Omega_{s} \backslash E \Longrightarrow & w_{s}^{(k)}(x) \in\left[\underline{m}(v)+\sigma_{k}, \underline{m}(v) \mid+k\right], \\
\left\|w_{s}^{(k)}-q_{s} v\right\|_{L^{p}\left(\Omega_{s}\right) \leqslant} & 2\left\|v_{s}-q_{s} v\right\|_{L^{p}\left(\Omega_{s}\right)} \\
& +4 \sigma_{k}(\operatorname{meas} \Omega)^{1 / p}+2\left(\int_{E_{s}^{(k)}}|v|^{p} \mathrm{~d} x\right)^{1 / p} \\
\left(\operatorname{meas} E_{s}^{(k)}\right)^{1 / p} \leqslant & 2 \sigma_{k}^{-1}\left\|v_{s}-q_{s} v\right\|_{L^{p}\left(\Omega_{s}\right)} \\
& +k^{-1}\left\{2(\operatorname{meas} \Omega)^{1 / p}+\|v\|_{L^{p}(\Omega)}\right\} .
\end{aligned}
$$


By virtue of condition $(\bar{*})$ and relations (3.51), (3.33), and (3.58), there exists an increasing sequence $\left\{s_{k}\right\} \subset \mathbb{N}$ such that, for every $k \in \mathbb{N}$ and every $s \in \mathbb{N}, s \geqslant s_{k}$,

$$
\begin{aligned}
& {\left[\underline{m}(v)+\sigma_{k},|\underline{m}(v)|+k\right] \subset \Phi\left(h_{s}\right),} \\
& \left(\operatorname{meas} E_{s}^{(k)}\right)^{1 / p} \leqslant 2 k^{-1}\left\{(\operatorname{meas} \Omega)^{1 / p}+\|v\|_{L^{p}(\Omega)}\right\} .
\end{aligned}
$$

Using the functions $w_{s}^{(k)}$, the sets $E_{s}^{(k)}$, and the sequence $\left\{s_{k}\right\}$, we define the functions $w_{s}$ and the sets $E_{s}$ in the same way as in case (i). In addition, let $\left\{\tilde{E}_{s}\right\}$ be the sequence of sets such that

$$
\tilde{E}_{s}=\left\{\begin{array}{l}
\tilde{E}_{s}^{(1)} \text { if } s \leqslant s_{1}, \\
\tilde{E}_{s}^{(k)} \text { if } s_{k}<s \leqslant s_{k+1}, k \in \mathbb{N} .
\end{array}\right.
$$

For every $s \in \mathbb{N}$, we set $\beta_{s}=1$. Finally, we define $\tilde{s}=s_{1}+1$.

Obviously, for every $s \in \mathbb{N}$, we have $w_{s} \in W^{1, p}\left(\Omega_{s}\right)$. Moreover, for every $s \in \mathbb{N}$, the sets $E_{s}$ and $\tilde{E}_{s}$ are contained in $\Omega_{s}$ and are measurable. It is also clear that $\left\{\beta_{s}\right\} \subset(0,1]$ and $\tilde{s} \in \mathbb{N}$. Using (3.60), we establish that meas $E_{s} \rightarrow 0$. This along with (3.33), (3.37), and (3.57) implies that $\left\|w_{s}-q_{s} v\right\|_{L^{p}\left(\Omega_{s}\right)} \rightarrow 0$. Thus, assertions (a) and (d) hold. In view of (3.55), assertion (b) holds. From (3.56) and (3.59), we derive that assertion (c) holds. Finally, assertions (e) and (f) are obvious. Thus, in case (ii), the conclusion of the proposition holds.

Case (iii) is considered similarly to case (ii). We only note that to obtain the required conclusion in case (iii), the following functions and sets are used for every $k, s \in \mathbb{N}$ :

$$
\begin{aligned}
v_{s}^{(k)} & =\min \left\{v_{s}, q_{s} v+\sigma_{k}\right\}-2 \sigma_{k}, \quad w_{s}^{(k)}=\max \left\{v_{s}^{(k)},-|\bar{m}(v)|-k\right\}, \\
E_{s}^{(k)} & =\left\{v_{s} \geqslant q_{s} v+\sigma_{k}\right\} \cup\left\{v_{s}^{(k)} \leqslant-|\bar{m}(v)|-k\right\}, \\
\tilde{E}_{s}^{(k)} & =\left\{v_{s}^{(k)} \leqslant-|\bar{m}(v)|-k\right\} .
\end{aligned}
$$

Here, as in case (ii), $\left\{\sigma_{k}\right\} \subset(0,1)$ and $\sigma_{k} \rightarrow 0$.

Finally, let us consider case (iv). By virtue of Proposition 3.2, we have

$$
(-\infty,+\infty) \subset \Phi(h) .
$$

For every $k, s \in \mathbb{N}$, we set

$$
w_{s}^{(k)}=\min \left\{\max \left\{v_{s},-k\right\}, k\right\}, \quad E_{s}^{(k)}=\left\{\left|v_{s}\right| \geqslant k\right\} .
$$

It is not difficult to see that if $k, s \in \mathbb{N}$, then $w_{s}^{(k)} \in W^{1, p}\left(\Omega_{s}\right)$,

$$
\begin{aligned}
-k & \leqslant w_{s}^{(k)} \leqslant k \text { in } \Omega_{s}, \\
\left\|w_{s}^{(k)}-q_{s} v\right\|_{L^{p}\left(\Omega_{s}\right)} & \leqslant 2\left\|v_{s}-q_{s} v\right\|_{L^{p}\left(\Omega_{s}\right)}+2\left(\int_{E_{s}^{(k)}}|v|^{p} \mathrm{~d} x\right)^{1 / p}, \\
\nabla w_{s}^{(k)} & =\nabla v_{s} \cdot 1_{\Omega_{s} \backslash E_{s}^{(k)}} \text { a.e. in } \Omega_{s}, \\
\left(\operatorname{meas} E_{s}^{(k)}\right)^{1 / p} & \leqslant k^{-1}\left\{\left\|v_{s}-q_{s} v\right\|_{L^{p}\left(\Omega_{s}\right)}+\|v\|_{L^{p}(\Omega)}\right\} .
\end{aligned}
$$

By virtue of condition ( $\bar{*}$ ) and relations (3.61), (3.33), and (3.65), there exists an increasing sequence $\left\{s_{k}\right\} \subset \mathbb{N}$ such that, for every $k \in \mathbb{N}$ and every $s \in \mathbb{N}, s \geqslant s_{k}$,

$$
\begin{aligned}
& {[-k, k] \subset \Phi\left(h_{s}\right),} \\
& \text { meas } E_{s}^{(k)} \leqslant 2 k^{-p}\|v\|_{L^{p}(\Omega)}^{p} .
\end{aligned}
$$


Using the functions $w_{s}^{(k)}$, the sets $E_{s}^{(k)}$, and the sequence $\left\{s_{k}\right\}$, we define the functions $w_{s}$ and the sets $E_{s}$ in the same way as in case (i). Moreover, for every $s \in \mathbb{N}$, we set $\tilde{E}_{s}=E_{s}$ and $\beta_{s}=1$. Finally, we define $\tilde{s}=s_{1}+1$.

Obviously, for every $s \in \mathbb{N}$, we have $w_{s} \in W^{1, p}\left(\Omega_{s}\right)$. Moreover, for every $s \in \mathbb{N}$, the sets $E_{s}$ and $\tilde{E}_{s}$ are contained in $\Omega_{s}$ and are measurable. It is also clear that $\left\{\beta_{s}\right\} \subset(0,1]$ and $\tilde{s} \in \mathbb{N}$. Using (3.67), we find that meas $E_{s} \rightarrow 0$. This along with (3.33) and (3.63) implies that $\left\|w_{s}-q_{s} v\right\|_{L^{p}\left(\Omega_{s}\right)} \rightarrow 0$. Thus, assertions (a) and (d) hold. In view of (3.64), assertion (b) holds. From (3.62) and (3.66), we derive that assertion (c) holds. Finally, assertions (e) and (f) are obvious. Thus, in case (iv), the conclusion of the proposition holds.

Proposition 3.7 Assume that the embedding of $W^{1, p}(\Omega)$ into $L^{p}(\Omega)$ is compact. Let, for every $s \in \mathbb{N}, h_{s}: \mathbb{R} \rightarrow \mathbb{R}$. Let $h: \mathbb{R} \rightarrow \mathbb{R}$ be a function such that the set $\Phi(h)$ is nonempty and closed. Assume that the following conditions are satisfied:

$\left(*^{\prime}\right)$ if $t \in \Phi(h)$, then there exist $t_{1}, t_{2} \in \mathbb{R}$ such that $t_{1}<t_{2}$ and $t \in\left[t_{1}, t_{2}\right] \subset \Phi(h)$;

$\left(*^{\prime \prime}\right)$ if $t_{1}, t_{2} \in \mathbb{R}, t_{1}<t_{2},\left(t_{1}, t_{2}\right) \subset \Phi(h)$, and $0<\sigma<\left(t_{2}-t_{1}\right) / 2$, then there exists $\bar{s} \in \mathbb{N}$ such that, for every $s \in \mathbb{N}, s \geqslant \bar{s}$, we have $\left[t_{1}+\sigma, t_{2}-\sigma\right] \subset \Phi\left(h_{s}\right)$.

Let $v \in U(h)$, and let $\underline{m}(v)=\bar{m}(v)$. Let, for every $s \in \mathbb{N}, v_{s} \in W^{1, p}\left(\Omega_{s}\right)$. Assume that $\left\|v_{s}-q_{s} v\right\|_{L^{p}\left(\Omega_{s}\right)} \rightarrow 0$.

Then there exist a sequence of functions $w_{s} \in W^{1, p}\left(\Omega_{s}\right)$, a sequence of measurable sets $E_{s} \subset \Omega_{s}$, and a number $\tilde{s} \in \mathbb{N}$ such that the following assertions hold:

(a) $\left\|w_{s}-q_{s} v\right\|_{L^{p}\left(\Omega_{s}\right)} \rightarrow 0$;

(b) for every $s \in \mathbb{N}, \nabla w_{s}=\nabla v_{s} \cdot 1_{\Omega_{s} \backslash E_{s}}$ a.e. in $\Omega_{s}$;

(c) for every $s \in \mathbb{N}, s \geqslant \tilde{s}$, we have $w_{s} \in U_{s}\left(h_{s}\right)$;

(d) meas $E_{s} \rightarrow 0$.

Proof Since $\underline{m}(v)=\bar{m}(v)$, we have $\underline{m}(v) \in \mathbb{R}$ and $v=\underline{m}(v)$ a.e. in $\Omega$. In turn, in view of the inclusion $\underline{m}(v) \in \mathbb{R}$, we derive from Proposition 3.2 that $\underline{m}(v) \in \Phi(h)$. Then, by virtue of condition $\left(*^{\prime}\right)$, there exist $a_{1}, a_{2} \in \mathbb{R}$ such that

$$
a_{1}<a_{2}, \quad \underline{m}(v) \in\left[a_{1}, a_{2}\right] \subset \Phi(h) .
$$

We set

$$
a_{*}= \begin{cases}1 & \text { if } \underline{m}(v)=a_{1}, \\ 0 & \text { if } a_{1}<\underline{m}(v)<a_{2}, \\ -1 & \text { if } \underline{m}(v)=a_{2}\end{cases}
$$

and

$$
\sigma_{*}=\frac{1}{4}\left|a_{*}\right|\left(a_{2}-a_{1}\right)+\frac{1}{2} \min \left\{\underline{m}(v)-a_{1}, a_{2}-\underline{m}(v)\right\} .
$$

It is easy to see that $\sigma_{*}>0$. We fix a sequence $\left\{\sigma_{k}\right\} \subset\left(0, \sigma_{*}\right)$ such that $\sigma_{k} \rightarrow 0$.

For every $k, s \in \mathbb{N}$, we set

$$
\begin{aligned}
& w_{s}^{(k)}=\min \left\{\max \left\{v_{s}, \underline{m}(v)-\sigma_{k}\right\}, \underline{m}(v)+\sigma_{k}\right\}+2 a_{*} \sigma_{k}, \\
& E_{s}^{(k)}=\left\{\left|v_{s}-\underline{m}(v)\right| \geqslant \sigma_{k}\right\} .
\end{aligned}
$$


If $k, s \in \mathbb{N}$, then we have $w_{s}^{(k)} \in W^{1, p}\left(\Omega_{S}\right)$,

$$
\begin{aligned}
a_{1}+\sigma_{k} \leqslant w_{s}^{(k)} & \leqslant a_{2}-\sigma_{k} \text { in } \Omega_{s}, \\
\left\|w_{s}^{(k)}-q_{s} v\right\|_{L^{p}\left(\Omega_{s}\right)} & \leqslant\left\|v_{s}-q_{s} v\right\|_{L^{p}\left(\Omega_{s}\right)}+5 \sigma_{k}(\text { meas } \Omega)^{1 / p}, \\
\nabla w_{s}^{(k)} & =\nabla v_{s} \cdot 1_{\Omega_{s} \backslash E_{s}^{(k)}} \text { a.e. in } \Omega_{s}, \\
\text { meas } E_{s}^{(k)} & \leqslant \sigma_{k}^{-p}\left\|v_{s}-q_{s} v\right\|_{L^{p}\left(\Omega_{s}\right)}^{p} .
\end{aligned}
$$

Owing to relations (3.68), the inclusion $\left\{\sigma_{k}\right\} \subset\left(0, \sigma_{*}\right)$, condition $\left(*^{\prime \prime}\right)$, inequality (3.72), and the fact that $\left\|v_{s}-q_{s} v\right\|_{L^{p}\left(\Omega_{s}\right)} \rightarrow 0$, there exists an increasing sequence $\left\{s_{k}\right\} \subset \mathbb{N}$ such that, for every $k \in \mathbb{N}$ and every $s \in \mathbb{N}, s \geqslant s_{k}$,

$$
\left[a_{1}+\sigma_{k}, a_{2}-\sigma_{k}\right] \subset \Phi\left(h_{s}\right), \quad \text { meas } E_{s}^{(k)} \leqslant \sigma_{k} .
$$

Using the functions $w_{s}^{(k)}$, the sets $E_{s}^{(k)}$, and the sequence $\left\{s_{k}\right\}$, we define the functions $w_{s}$ and the sets $E_{s}$ in the same way as in case (i) in the proof of Proposition 3.6. In addition, we define $\tilde{s}=s_{1}+1$.

Obviously, for every $s \in \mathbb{N}$, we have $w_{s} \in W^{1, p}\left(\Omega_{s}\right)$. Moreover, for every $s \in \mathbb{N}$, the set $E_{s}$ is contained in $\Omega_{s}$ and is measurable. Clearly, $\tilde{s} \in \mathbb{N}$. Since $\left\|v_{s}-q_{s} v\right\|_{L^{p}\left(\Omega_{s}\right)} \rightarrow 0$ and $\sigma_{k} \rightarrow 0$, we derive from (3.70) that assertion (a) holds. In view of (3.71), assertion (b) holds. Using (3.69) and the first relation in (3.73), we establish that assertion (c) holds. Finally, since $\sigma_{k} \rightarrow 0$, we derive from the second relation in (3.73) that assertion (d) holds.

\section{Main results}

We assume that the following conditions are satisfied:

$\left(*_{1}\right)$ the embedding of $W^{1, p}(\Omega)$ into $L^{p}(\Omega)$ is compact;

$\left(*_{2}\right)$ the sequence of spaces $W^{1, p}\left(\Omega_{S}\right)$ is strongly connected with the space $W^{1, p}(\Omega)$;

$\left(*_{3}\right)$ there exists a functional $F: W^{1, p}(\Omega) \rightarrow \mathbb{R}$ such that the sequence $\left\{F_{S}\right\} \Gamma$-converges to the functional $F$;

$\left(*_{4}\right)$ there exists a functional $G: W^{1, p}(\Omega) \rightarrow \mathbb{R}$ such that for every function $v \in W^{1, p}(\Omega)$ and every sequence $v_{s} \in W^{1, p}\left(\Omega_{s}\right)$ with the property $\left\|v_{s}-q_{s} v\right\|_{L^{p}\left(\Omega_{s}\right)} \rightarrow 0$, we have $G_{s}\left(v_{s}\right) \rightarrow G(v)$.

These conditions will be essentially used in the proof of our main results.

Theorem 4.1 Assume that the following condition is satisfied:

(*) for every sequence of measurable sets $K_{s} \subset \Omega_{s}$ such that meas $K_{s} \rightarrow 0$, we have $\int_{K_{s}} \mu_{s} d x \rightarrow 0$

Let, for every $s \in \mathbb{N}, h_{s}: \mathbb{R} \rightarrow \mathbb{R}$ be a function such that the set $\Phi\left(h_{s}\right)$ is nonempty and closed. Let $h: \mathbb{R} \rightarrow \mathbb{R}$ be a function such that the set $\Phi(h)$ is nonempty and closed. Assume that the following conditions are satisfied:

$\left(*^{\prime}\right)$ if $t \in \Phi(h)$, then there exist $t_{1}, t_{2} \in \mathbb{R}$ such that $t_{1}<t_{2}$ and $t \in\left[t_{1}, t_{2}\right] \subset \Phi(h)$;

$\left(*^{\prime \prime}\right)$ if $t_{1}, t_{2} \in \mathbb{R}, t_{1}<t_{2},\left(t_{1}, t_{2}\right) \subset \Phi(h)$, and $0<\sigma<\left(t_{2}-t_{1}\right) / 2$, then there exists $\bar{s} \in \mathbb{N}$ such that, for every $s \in \mathbb{N}$, $s \geqslant \bar{s}$, we have $\left[t_{1}+\sigma, t_{2}-\sigma\right] \subset \Phi\left(h_{s}\right)$;

$\left(*^{\prime \prime \prime}\right)$ if $t_{s} \rightarrow t$ in $\mathbb{R},\left\{\tilde{s}_{j}\right\}$ is an increasing sequence in $\mathbb{N}$, and, for every $j \in \mathbb{N}$, we have $t_{\tilde{s}_{j}} \in \Phi\left(h_{\tilde{s}_{j}}\right)$, then $t \in \Phi(h)$. 
Let, for every $s \in \mathbb{N}$, $u_{s}$ be a function in $U_{s}\left(h_{s}\right)$ minimizing the functional $F_{s}+G_{s}$ on the set $U_{s}\left(h_{s}\right)$.

Then there exist an increasing sequence $\left\{s_{j}\right\} \subset \mathbb{N}$ and a function $u \in U(h)$ such that the following assertions hold:

(a) the function u minimizes the functional $F+G$ on the set $U(h)$;

(b) $\left\|u_{s_{j}}-q_{s_{j}} u\right\|_{L^{p}\left(\Omega_{s_{j}}\right)} \rightarrow 0$;

(c) $\left(F_{s_{j}}+G_{s_{j}}\right)\left(u_{s_{j}}\right) \rightarrow(F+G)(u)$.

Proof First, we show that the sequence of norms $\left\|u_{s}\right\|_{W^{1, p}\left(\Omega_{s}\right)}$ is bounded. Indeed, owing to the nonemptiness of the set $\Phi(h)$ and condition $\left(*^{\prime}\right)$, there exist $t_{1}, t_{2} \in \mathbb{R}$ such that $t_{1}<t_{2}$ and $\left(t_{1}, t_{2}\right) \subset \Phi(h)$. We set $\bar{t}=\left(t_{1}+t_{2}\right) / 2$. Let, for every $s \in \mathbb{N}, \varphi_{s}: \Omega_{s} \rightarrow \mathbb{R}$ be the function such that, for every $x \in \Omega_{s}, \varphi_{s}(x)=\bar{t}$. Obviously, if $s \in \mathbb{N}$, then $\varphi_{s} \in W^{1, p}\left(\Omega_{s}\right)$. In view of condition $\left(*_{4}\right)$, the sequence $\left\{G_{s}\left(\varphi_{s}\right)\right\}$ is bounded. This along with $(2.1)$ and the boundedness of the sequence of norms $\left\|\mu_{S}\right\|_{L^{1}\left(\Omega_{s}\right)}$ implies that there exists a positive constant $M$ such that

$$
\forall s \in \mathbb{N}, \quad\left(F_{s}+G_{s}\right)\left(\varphi_{s}\right) \leqslant M .
$$

Moreover, since $t_{1}<t_{2}$ and $\left(t_{1}, t_{2}\right) \subset \Phi(h)$, by virtue of condition $\left(*^{\prime \prime}\right)$, there exists $\bar{s} \in \mathbb{N}$ such that, for every $s \in \mathbb{N}, s \geqslant \bar{s}$, we have $\bar{t} \in \Phi\left(h_{s}\right)$. Therefore, fixing $s \in \mathbb{N}, s \geqslant \bar{s}$, we have $\varphi_{s} \in U_{s}\left(h_{s}\right)$. Then, taking into account that the function $u_{s}$ minimizes the functional $F_{s}+G_{s}$ on the set $U_{s}\left(h_{s}\right)$, we obtain the inequality $\left(F_{s}+G_{s}\right)\left(u_{s}\right) \leqslant\left(F_{s}+G_{s}\right)\left(\varphi_{s}\right)$. This inequality along with (2.3) and (4.1) yields the estimate $\left\|u_{s}\right\|_{W^{1, p}\left(\Omega_{s}\right)}^{p} \leqslant\left(M+c_{6}\right) / c_{5}$. Hence, we conclude that the sequence of norms $\left\|u_{s}\right\|_{W^{1, p}\left(\Omega_{s}\right)}$ is bounded.

Next, since conditions $\left(*_{1}\right),\left(*_{2}\right)$, and $\left(*^{\prime \prime \prime}\right)$ are satisfied, taking into account the inclusions $u_{s} \in U_{s}\left(h_{s}\right), s \in \mathbb{N}$, and the boundedness of the sequence of norms $\left\|u_{s}\right\|_{W^{1, p}\left(\Omega_{s}\right)}$, we derive from Proposition 3.5 that there exist an increasing sequence $\left\{s_{j}\right\} \subset \mathbb{N}$ and a function $u \in U(h)$ such that

$$
\left\|u_{s_{j}}-q_{s_{j}} u\right\|_{L^{p}\left(\Omega_{s_{j}}\right)} \rightarrow 0 .
$$

We define the sequence $\left\{\bar{u}_{s}\right\}$ as follows:

$$
\bar{u}_{s}=\left\{\begin{array}{l}
u_{s} \text { if } s=s_{j} \text { for some } j \in \mathbb{N} \\
q_{s} u \text { if } s \neq s_{j} \text { for every } j \in \mathbb{N}
\end{array}\right.
$$

Obviously, for every $s \in \mathbb{N}, \bar{u}_{s} \in W^{1, p}\left(\Omega_{s}\right)$. It follows from (4.2) that

$$
\left\|\bar{u}_{s}-q_{s} u\right\|_{L^{p}\left(\Omega_{s}\right)} \rightarrow 0 .
$$

Then, by virtue of condition $\left(*_{3}\right)$, we have

$$
\liminf _{s \rightarrow \infty} F_{S}\left(\bar{u}_{s}\right) \geqslant F(u) .
$$

In addition, we infer from (4.3) and condition $\left(*_{4}\right)$ that $G_{s}\left(\bar{u}_{s}\right) \rightarrow G(u)$. This and (4.4) imply that

$$
\liminf _{j \rightarrow \infty}\left(F_{s_{j}}+G_{s_{j}}\right)\left(u_{s_{j}}\right) \geqslant(F+G)(u) .
$$

Further, we fix an arbitrary $v \in U(h)$. In view of condition $\left(*_{3}\right)$, there exists a sequence $v_{s} \in W^{1, p}\left(\Omega_{s}\right)$ such that

$$
\begin{aligned}
\left\|v_{s}-q_{s} v\right\|_{L^{p}\left(\Omega_{s}\right)} & \rightarrow 0, \\
F_{S}\left(v_{s}\right) & \rightarrow F(v) .
\end{aligned}
$$


Assume that $\underline{m}(v)<\bar{m}(v)$. Then, taking into account the limit relation (4.6) and conditions $\left(*_{1}\right),\left(*^{\prime}\right)$, and $\left(*^{\prime \prime}\right)$, we derive from Proposition 3.6 that there exist a sequence of functions $w_{s} \in W^{1, p}\left(\Omega_{s}\right)$, sequences of measurable sets $E_{s} \subset \Omega_{s}$ and $\tilde{E}_{s} \subset \Omega_{s}$, a sequence $\left\{\beta_{s}\right\} \subset(0,1]$, and a number $\tilde{s} \in \mathbb{N}$ such that assertions (a)-(f) in the conclusion of Proposition 3.6 hold. Owing to assertion (a) in the conclusion of Proposition 3.6 and to condition $\left(*_{4}\right)$, we have

$$
G_{s}\left(w_{s}\right) \rightarrow G(v) .
$$

Moreover, $F_{S}\left(w_{s}\right) \rightarrow F(v)$. Indeed, using assertions (b) and (e) in the conclusion of Proposition 3.6, the convexity of the functions $f_{s}(x, \cdot)$ for a.e. $x \in \Omega_{s}, s \in \mathbb{N}$, and (2.1), we find that, for every $s \in \mathbb{N}$,

$$
F_{S}\left(w_{s}\right) \leqslant F_{S}\left(v_{s}\right)+2\left(1-\beta_{S}\right)\left\|\mu_{s}\right\|_{L^{1}\left(\Omega_{s}\right)}+2 \int_{E_{s}} \mu_{s} \mathrm{~d} x+c_{2} \int_{E_{s}}|\nabla v|^{p} \mathrm{~d} x .
$$

Hence, taking into account the limit relation (4.7), assertions (d) and (f) in the conclusion of Proposition 3.6, and condition (*), we establish that the upper limit of the sequence $\left\{F_{s}\left(w_{s}\right)\right\}$ is not greater than $F(v)$. In addition, due to assertion (a) in the conclusion of Proposition 3.6 and to condition $\left(*_{3}\right)$, the lower limit of the sequence $\left\{F_{s}\left(w_{s}\right)\right\}$ is not less than $F(v)$. Thus, $F_{S}\left(w_{s}\right) \rightarrow F(v)$. This and (4.8) imply that

$$
\left(F_{s}+G_{s}\right)\left(w_{s}\right) \rightarrow(F+G)(v) .
$$

Moreover, using assertion (c) in the conclusion of Proposition 3.6 and the fact that, for every $s \in \mathbb{N}$, the function $u_{s}$ minimizes the functional $F_{s}+G_{s}$ on the set $U_{s}\left(h_{s}\right)$, we find that $\left(F_{s}+G_{s}\right)\left(u_{s}\right) \leqslant\left(F_{s}+G_{s}\right)\left(w_{s}\right)$ for every $s \in \mathbb{N}, s \geqslant \tilde{s}$. This along with (4.9) yields the inequality

$$
\limsup _{s \rightarrow \infty}\left(F_{s}+G_{s}\right)\left(u_{s}\right) \leqslant(F+G)(v) .
$$

Now, let $\underline{m}(v)=\bar{m}(v)$. Then, taking into account the limit relation (4.6) and conditions $\left(*_{1}\right),\left(*^{\prime}\right)$, and $\left(*^{\prime \prime}\right)$, we derive from Proposition 3.7 that there exist a sequence of functions $w_{s} \in W^{1, p}\left(\Omega_{s}\right)$, a sequence of measurable sets $E_{s} \subset \Omega_{s}$, and a number $\tilde{s} \in \mathbb{N}$ such that assertions (a)-(d) in the conclusion of Proposition 3.7 hold. Using assertion (b) in the conclusion of Proposition 3.7 and (2.1), we find that, for every $s \in \mathbb{N}$,

$$
F_{S}\left(w_{s}\right) \leqslant F_{S}\left(v_{s}\right)+2 \int_{E_{S}} \mu_{S} \mathrm{~d} x .
$$

Hence, taking into account the limit relation (4.7), assertion (d) in the conclusion of Proposition 3.7, and condition (*), we establish that the upper limit of the sequence $\left\{F_{S}\left(w_{s}\right)\right\}$ is not greater than $F(v)$. In addition, due to assertion (a) in the conclusion of Proposition 3.7 and to condition $\left(*_{3}\right)$, the lower limit of the sequence $\left\{F_{s}\left(w_{s}\right)\right\}$ is not less than $F(v)$. Thus, $F_{s}\left(w_{s}\right) \rightarrow F(v)$. Moreover, owing to assertion (a) in the conclusion of Proposition 3.7 and to condition (*4), we have $G_{s}\left(w_{s}\right) \rightarrow G(v)$. In sum, $\left(F_{s}+G_{s}\right)\left(w_{s}\right) \rightarrow(F+G)(v)$. This along with assertion (c) in the conclusion of Proposition 3.7 and the fact that, for every $s \in \mathbb{N}$, the function $u_{s}$ minimizes the functional $F_{s}+G_{s}$ on the set $U_{s}\left(h_{s}\right)$ leads us to inequality (4.10).

Thus, inequality (4.10) holds for both possible relations between $\underline{m}(v)$ and $\bar{m}(v)$. Taking into account the arbitrariness of the function $v \in U(h)$, we derive from (4.5) and (4.10) that the function $u$ minimizes the functional $F+G$ on the set $U(h)$. Finally, inequality (4.5) and inequality (4.10) with $v=u$ imply that $\left(F_{s_{j}}+G_{s_{j}}\right)\left(u_{s_{j}}\right) \rightarrow(F+G)(u)$.

Theorem 4.2 Assume that

$$
\left\|\mu_{s}\right\|_{L^{1}\left(\Omega_{s}\right)} \rightarrow 0
$$


Let, for every $s \in \mathbb{N}, h_{s}: \mathbb{R} \rightarrow \mathbb{R}$ be a function such that the set $\Phi\left(h_{s}\right)$ is nonempty and closed. Let $h: \mathbb{R} \rightarrow \mathbb{R}$ be a function such that the set $\Phi(h)$ is closed and has nonempty interior. Assume that the following conditions are satisfied:

$\left(*^{\prime}\right)$ if $t \in \Phi(h)$, then there exists a sequence $\left\{t_{s}\right\} \subset \mathbb{R}$ such that $t_{s} \rightarrow t$ and, for every $s \in \mathbb{N}, t_{s} \in \Phi\left(h_{s}\right)$;

$\left(*^{\prime \prime}\right)$ if $t_{1}, t_{2} \in \mathbb{R}, t_{1}<t_{2},\left(t_{1}, t_{2}\right) \subset \Phi(h)$, and $0<\sigma<\left(t_{2}-t_{1}\right) / 2$, then there exists $\bar{s} \in \mathbb{N}$ such that, for every $s \in \mathbb{N}, s \geqslant \bar{s}$, we have $\left[t_{1}+\sigma, t_{2}-\sigma\right] \subset \Phi\left(h_{s}\right)$;

$\left(*^{\prime \prime \prime}\right)$ if $t_{s} \rightarrow t$ in $\mathbb{R},\left\{\tilde{s}_{j}\right\}$ is an increasing sequence in $\mathbb{N}$, and, for every $j \in \mathbb{N}$, we have $t_{\tilde{s}_{j}} \in \Phi\left(h_{\tilde{s}_{j}}\right)$, then $t \in \Phi(h)$.

Let, for every $s \in \mathbb{N}, u_{s}$ be a function in $U_{s}\left(h_{s}\right)$ minimizing the functional $F_{s}+G_{s}$ on the set $U_{s}\left(h_{s}\right)$.

Then there exist an increasing sequence $\left\{s_{j}\right\} \subset \mathbb{N}$ and a function $u \in U(h)$ such that the following assertions hold:

(a) the function $u$ minimizes the functional $F+G$ on the set $U(h)$;

(b) $\left\|u_{s_{j}}-q_{s_{j}} u\right\|_{L^{p}\left(\Omega_{s_{j}}\right)} \rightarrow 0$;

(c) $\left(F_{s_{j}}+G_{s_{j}}\right)\left(u_{s_{j}}\right) \rightarrow(F+G)(u)$.

Proof First, we show that the sequence of norms $\left\|u_{s}\right\|_{W^{1, p}\left(\Omega_{s}\right)}$ is bounded. We fix $t \in \Phi(h)$. By virtue of condition $\left(*^{\prime}\right)$, there exists a sequence $\left\{t_{s}\right\} \subset \mathbb{R}$ such that $t_{s} \rightarrow t$ and

$$
\forall s \in \mathbb{N}, \quad t_{s} \in \Phi\left(h_{s}\right) .
$$

Let $\varphi: \Omega \rightarrow \mathbb{R}$ be the function such that, for every $x \in \Omega, \varphi(x)=t$. It is clear that $\varphi \in W^{1, p}(\Omega)$. Let, for every $s \in \mathbb{N}, \varphi_{s}: \Omega_{s} \rightarrow \mathbb{R}$ be the function such that, for every $x \in \Omega_{s}, \varphi_{s}(x)=t_{s}$. Obviously, if $s \in \mathbb{N}$, then $\varphi_{s} \in W^{1, p}\left(\Omega_{s}\right)$. Furthermore, in view of (4.12), we have $\varphi_{s} \in U_{s}\left(h_{s}\right)$ for every $s \in \mathbb{N}$. Then, taking into account that, for every $s \in \mathbb{N}$, the function $u_{s}$ minimizes the functional $F_{s}+G_{s}$ on the set $U_{s}\left(h_{s}\right)$, we find that, for every $s \in \mathbb{N},\left(F_{s}+G_{s}\right)\left(u_{s}\right) \leqslant\left(F_{s}+G_{s}\right)\left(\varphi_{s}\right)$. This and (2.3) imply that, for every $s \in \mathbb{N}$,

$$
c_{5}\left\|u_{s}\right\|_{W^{1, p}\left(\Omega_{s}\right)}^{p} \leqslant\left(F_{s}+G_{s}\right)\left(\varphi_{s}\right)+c_{6} .
$$

Owing to (2.1) and to the boundedness of the sequence of norms $\left\|\mu_{s}\right\|_{L^{1}\left(\Omega_{s}\right)}$, the sequence $\left\{F_{s}\left(\varphi_{s}\right)\right\}$ is bounded. Moreover, since $t_{s} \rightarrow t$, we have $\left\|\varphi_{s}-q_{s} \varphi\right\|_{L^{p}\left(\Omega_{s}\right)} \rightarrow 0$. Then, in view of condition $\left(*_{4}\right), G_{s}\left(\varphi_{s}\right) \rightarrow G(\varphi)$. Consequently, the sequence $\left\{G_{s}\left(\varphi_{s}\right)\right\}$ is bounded. From (4.13) and the boundedness of the sequences $\left\{F_{S}\left(\varphi_{S}\right)\right\}$ and $\left\{G_{S}\left(\varphi_{S}\right)\right\}$, we derive that the sequence of norms $\left\|u_{s}\right\|_{W^{1, p}\left(\Omega_{s}\right)}$ is bounded.

Now, since conditions $\left(*_{1}\right),\left(*_{2}\right)$, and $\left(*^{\prime \prime \prime}\right)$ are satisfied, we infer from Proposition 3.5 that there exist an increasing sequence $\left\{s_{j}\right\} \subset \mathbb{N}$ and a function $u \in U(h)$ such that $\| u_{s_{j}}-$ $q_{s_{j}} u \|_{L^{p}\left(\Omega_{s_{j}}\right)} \rightarrow 0$. This along with conditions $\left(*_{3}\right)$ and $\left(*_{4}\right)$ implies inequality (4.5).

Further, we fix an arbitrary $v \in U(h)$. In view of condition $\left(*_{3}\right)$, there exists a sequence $v_{s} \in W^{1, p}\left(\Omega_{S}\right)$ such that the limit relations (4.6) and (4.7) hold.

Assume that $\underline{m}(v)<\bar{m}(v)$. Then, taking into account the limit relation (4.6) and conditions $\left(*_{1}\right)$ and $\left(*^{\prime \prime}\right)$, we deduce from Proposition 3.6 that there exist a sequence of functions $w_{s} \in W^{1, p}\left(\Omega_{s}\right)$, sequences of measurable sets $E_{s} \subset \Omega_{s}$ and $\tilde{E}_{s} \subset \Omega_{s}$, a sequence $\left\{\beta_{s}\right\} \subset(0,1]$, and a number $\tilde{s} \in \mathbb{N}$ such that assertions (a)-(f) in the conclusion of Proposition 3.6 hold. Using these assertions along with the limit relation (4.7) and conditions $\left(*_{3}\right)$, $\left(*_{4}\right)$, and (4.11) and arguing in the same way as in the corresponding place in the proof of Theorem 4.1, we obtain inequality (4.10). 
Now, let $\underline{m}(v)=\bar{m}(v)$. Then $\underline{m}(v) \in \mathbb{R}$ and $v=\underline{m}(v)$ a.e. in $\Omega$. This and the inclusion $v \in U(h)$ imply that $\underline{m}(v) \in \Phi(h)$. Then, by virtue of condition $\left(*^{\prime}\right)$, there exists a sequence $\left\{r_{s}\right\} \subset \mathbb{R}$ such that $r_{s} \rightarrow \underline{m}(v)$ and

$$
\forall s \in \mathbb{N}, \quad r_{s} \in \Phi\left(h_{s}\right) .
$$

Let, for every $s \in \mathbb{N}, y_{s}: \Omega_{s} \rightarrow \mathbb{R}$ be the function such that, for every $x \in \Omega_{s}, y_{s}(x)=r_{s}$. Obviously, if $s \in \mathbb{N}$, then $y_{s} \in W^{1, p}\left(\Omega_{s}\right)$. Moreover, since $v=\underline{m}(v)$ a.e. in $\Omega$ and $r_{s} \rightarrow$ $\underline{m}(v)$, we have $\left\|y_{s}-q_{s} v\right\|_{L^{p}\left(\Omega_{s}\right)} \rightarrow 0$. This along with conditions $\left(*_{3}\right)$ and $\left(*_{4}\right)$ yields

$$
\begin{aligned}
\liminf _{s \rightarrow \infty} F_{s}\left(y_{s}\right) & \geqslant F(v), \\
G_{s}\left(y_{s}\right) & \rightarrow G(v) .
\end{aligned}
$$

We note that, owing to (2.1), for every $s \in \mathbb{N}$, we have $\left|F_{S}\left(y_{s}\right)\right| \leqslant\left\|\mu_{s}\right\|_{L^{1}\left(\Omega_{s}\right)}$ and $F_{S}\left(v_{s}\right) \geqslant$ $-\left\|\mu_{s}\right\|_{L^{1}\left(\Omega_{s}\right)}$. Then, in view of (4.11) and (4.7), we have $F_{s}\left(y_{s}\right) \rightarrow 0$ and $F(v) \geqslant 0$. Using these relations and (4.15), we find that $F_{s}\left(y_{s}\right) \rightarrow F(v)$. This and (4.16) imply that

$$
\left(F_{s}+G_{s}\right)\left(y_{s}\right) \rightarrow(F+G)(v) .
$$

We also observe that, by virtue of (4.14), for every $s \in \mathbb{N}, y_{s} \in U_{s}\left(h_{s}\right)$. Then, taking into account that, for every $s \in \mathbb{N}$, the function $u_{s}$ minimizes the functional $F_{s}+G_{s}$ on the set $U_{s}\left(h_{s}\right)$, we establish that, for every $s \in \mathbb{N},\left(F_{s}+G_{s}\right)\left(u_{s}\right) \leqslant\left(F_{s}+G_{s}\right)\left(y_{s}\right)$. This and (4.17) yield inequality (4.10).

Thus, inequality (4.10) holds for both possible relations between $\underline{m}(v)$ and $\bar{m}(v)$. In view of the arbitrariness of the function $v \in U(h)$, we infer from (4.5) and (4.10) that the function $u$ minimizes the functional $F+G$ on the set $U(h)$. Finally, inequality (4.5) and inequality (4.10) with $v=u$ imply that $\left(F_{s_{j}}+G_{s_{j}}\right)\left(u_{s_{j}}\right) \rightarrow(F+G)(u)$.

Theorem 4.3 Assume that condition (4.11) is satisfied. Let, for every $s \in \mathbb{N}, h_{s}: \mathbb{R} \rightarrow \mathbb{R}$ be a function such that the set $\Phi\left(h_{s}\right)$ is nonempty and closed. Let $h: \mathbb{R} \rightarrow \mathbb{R}$ be a function such that the set $\Phi(h)$ is nonempty and closed and has empty interior. Assume that the following conditions are satisfied:

$\left(*^{\prime}\right)$ if $t \in \Phi(h)$, then there exists a sequence $\left\{t_{s}\right\} \subset \mathbb{R}$ such that $t_{s} \rightarrow t$ and, for every $s \in \mathbb{N}, t_{s} \in \Phi\left(h_{s}\right)$;

$\left(*^{\prime \prime}\right)$ if $t_{s} \rightarrow t$ in $\mathbb{R},\left\{\tilde{s}_{j}\right\}$ is an increasing sequence in $\mathbb{N}$, and, for every $j \in \mathbb{N}$, we have $t_{\tilde{s}_{j}} \in \Phi\left(h_{\tilde{s}_{j}}\right)$, then $t \in \Phi(h)$.

Let, for every $s \in \mathbb{N}, u_{s}$ be a function in $U_{s}\left(h_{s}\right)$ minimizing the functional $F_{s}+G_{s}$ on the set $U_{s}\left(h_{s}\right)$.

Then there exist an increasing sequence $\left\{s_{j}\right\} \subset \mathbb{N}$ and a function $u \in U(h)$ such that the following assertions hold:

(a) the function $u$ minimizes the functional $G$ on the set $U(h)$;

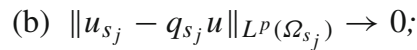

(c) $\left(F_{s_{j}}+G_{s_{j}}\right)\left(u_{s_{j}}\right) \rightarrow G(u)$.

Proof Arguing in the same way as in the proof of Theorem 4.2, we establish with the use of conditions $\left(*^{\prime}\right)$ and $\left(*_{4}\right)$ that the sequence of norms $\left\|u_{s}\right\|_{W^{1, p}\left(\Omega_{s}\right)}$ is bounded. Then, since conditions $\left(*_{1}\right),\left(*_{2}\right)$, and $\left(*^{\prime \prime}\right)$ are satisfied, we infer from Proposition 3.5 that there exist an increasing sequence $\left\{s_{j}\right\} \subset \mathbb{N}$ and a function $u \in U(h)$ such that $\left\|u_{s_{j}}-q_{s_{j}} u\right\|_{L^{p}\left(\Omega_{s_{j}}\right)} \rightarrow 0$. 
This along with condition $\left(*_{4}\right)$ implies that $G_{s_{j}}\left(u_{s_{j}}\right) \rightarrow G(u)$. Moreover, in view of (2.1) and (4.11), we have $\liminf _{s \rightarrow \infty} F_{s}\left(u_{s}\right) \geqslant 0$. Then

$$
\liminf _{j \rightarrow \infty}\left(F_{s_{j}}+G_{s_{j}}\right)\left(u_{s_{j}}\right) \geqslant G(u)
$$

Further, we fix an arbitrary $v \in U(h)$. Assume that $\underline{m}(v)<\bar{m}(v)$. Then, by virtue of Proposition 3.2, we have $(\underline{m}(v), \bar{m}(v)) \subset \Phi(h)$. However, this contradicts the fact that the set $\Phi(h)$ has empty interior. The obtained contradiction proves that $\underline{m}(v)=\bar{m}(v)$. Consequently, $\underline{m}(v) \in \mathbb{R}$ and $v=\underline{m}(v)$ a.e. in $\Omega$. This and the inclusion $v \in U(h)$ imply that $\underline{m}(v) \in \Phi(h)$. Then, by condition $\left(*^{\prime}\right)$, there exists a sequence $\left\{r_{s}\right\} \subset \mathbb{R}$ such that $r_{s} \rightarrow \underline{m}(v)$ and, for every $s \in \mathbb{N}, r_{s} \in \Phi\left(h_{s}\right)$. Let, for every $s \in \mathbb{N}, y_{s}: \Omega_{s} \rightarrow \mathbb{R}$ be the function such that, for every $x \in \Omega_{s}, y_{s}(x)=r_{s}$. It is clear that, for every $s \in \mathbb{N}, y_{s} \in U_{s}\left(h_{s}\right)$. Then, taking into account that, for every $s \in \mathbb{N}$, the function $u_{s}$ minimizes the functional $F_{s}+G_{s}$ on the set $U_{s}\left(h_{s}\right)$, we find that, for every $s \in \mathbb{N},\left(F_{s}+G_{s}\right)\left(u_{s}\right) \leqslant\left(F_{s}+G_{s}\right)\left(y_{s}\right)$. Since $v=\underline{m}(v)$ a.e. in $\Omega$ and $r_{s} \rightarrow \underline{m}(v)$, we have $\left\|y_{s}-q_{s} v\right\|_{L^{p}\left(\Omega_{s}\right)} \rightarrow 0$. This along with condition $\left(*_{4}\right)$ yields the relation $G_{s}\left(y_{s}\right) \rightarrow G(v)$. At the same time, in view of conditions (2.1) and (4.11), we have $F_{s}\left(y_{s}\right) \rightarrow 0$. Then $\left(F_{s}+G_{s}\right)\left(y_{s}\right) \rightarrow G(v)$ and, taking into account the above estimate for the numbers $\left(F_{s}+G_{s}\right)\left(u_{s}\right)$, we obtain the inequality $\limsup _{s \rightarrow \infty}\left(F_{s}+G_{s}\right)\left(u_{s}\right) \leqslant G(v)$. This along with (4.18) leads to the conclusion that the function $u$ minimizes the functional $G$ on the set $U(h)$ and $\left(F_{s_{j}}+G_{s_{j}}\right)\left(u_{s_{j}}\right) \rightarrow G(u)$.

Remark 4.4 Condition $\left(*_{3}\right)$ was not used in the proof of Theorem 4.3.

Remark 4.5 Theorems 4.1-4.3 can be applied to variational problems with various implicit constraints originally given in a form different from that of the constraints considered above. To see this, we introduce the following sets. For every function $h: \mathbb{R} \rightarrow \mathbb{R}$, we define

$$
\begin{aligned}
\Phi^{+}(h) & =\{t \in \mathbb{R}: h(t) \geqslant 0\}, \quad \Phi^{0}(h)=\{t \in \mathbb{R}: h(t)=0\}, \\
U^{+}(h) & =\left\{v \in W^{1, p}(\Omega): h(v) \geqslant 0 \text { a.e. in } \Omega\right\}, \\
U^{0}(h) & =\left\{v \in W^{1, p}(\Omega): h(v)=0 \text { a.e. in } \Omega\right\} .
\end{aligned}
$$

Moreover, for every $s \in \mathbb{N}$ and every function $h: \mathbb{R} \rightarrow \mathbb{R}$, we define

$$
\begin{gathered}
U_{s}^{+}(h)=\left\{v \in W^{1, p}\left(\Omega_{s}\right): h(v) \geqslant 0 \text { a.e. in } \Omega_{s}\right\}, \\
U_{s}^{0}(h)=\left\{v \in W^{1, p}\left(\Omega_{s}\right): h(v)=0 \text { a.e. in } \Omega_{s}\right\} .
\end{gathered}
$$

Using the introduced sets, we can formulate some results on the convergence of minimizers and minimum values of the functionals $F_{s}+G_{s}$ on the sets $U_{s}^{+}\left(h_{s}\right)$ and $U_{s}^{0}\left(h_{s}\right)$ for a given sequence of functions $h_{s}: \mathbb{R} \rightarrow \mathbb{R}$. These results are completely similar to Theorems 4.1-4.3. Without giving all possible statements, we note the following.

First, if, in the statements of Theorems 4.1-4.3, we replace the sets $\Phi\left(h_{s}\right), \Phi(h), U_{s}\left(h_{s}\right)$, and $U(h)$ by the sets $\Phi^{+}\left(h_{s}\right), \Phi^{+}(h), U_{s}^{+}\left(h_{s}\right)$, and $U^{+}(h)$, respectively, then we obtain the corresponding theorems on the convergence of minimizers and minimum values of the functionals $F_{s}+G_{s}$ on the sets $U_{s}^{+}\left(h_{s}\right)$.

Second, if, in the statements of Theorems 4.1-4.3, we replace the sets $\Phi\left(h_{s}\right), \Phi(h)$, $U_{s}\left(h_{s}\right)$, and $U(h)$ by the sets $\Phi^{0}\left(h_{s}\right), \Phi^{0}(h), U_{s}^{0}\left(h_{s}\right)$, and $U^{0}(h)$, respectively, then we obtain the corresponding theorems on the convergence of minimizers and minimum values of the functionals $F_{s}+G_{s}$ on the sets $U_{s}^{0}\left(h_{s}\right)$. 
To justify these assertions, it suffices to use Theorems 4.1-4.3 along with the following simple facts: if $h: \mathbb{R} \rightarrow \mathbb{R}$, then

$$
\begin{array}{ll}
\Phi^{+}(h)=\Phi(-h), & \Phi^{0}(h)=\Phi(|h|), \\
U^{+}(h)=U(-h), & U^{0}(h)=U(|h|) ;
\end{array}
$$

if $s \in \mathbb{N}$ and $h: \mathbb{R} \rightarrow \mathbb{R}$, then

$$
U_{s}^{+}(h)=U_{s}(-h), \quad U_{s}^{0}(h)=U_{s}(|h|) .
$$

Using Theorems 4.1-4.3, we can also obtain some results on the convergence of minimizers and minimum values of the functionals $F_{s}+G_{s}$ on the sets $U_{s}\left(h_{s}\right) \cap U_{s}\left(\bar{h}_{s}\right)$ for given sequences of functions $h_{s}, \bar{h}_{s}: \mathbb{R} \rightarrow \mathbb{R}$. These results are similar to the mentioned theorems. Without giving the corresponding statements, we note that they contain requirements on the sets $\Phi\left(\max \left\{h_{s}, \bar{h}_{s}\right\}\right)$ similar to the conditions of Theorems $4.1-4.3$ on the sets $\Phi\left(h_{s}\right)$. At the same time, these statements and, in particular, requirements involve a function $h: \mathbb{R} \rightarrow \mathbb{R}$ and the set $\Phi(h)$ related to the sets $\Phi\left(\max \left\{h_{s}, \bar{h}_{s}\right\}\right)$ in the same way as the corresponding sets are related in the statements of Theorems 4.1-4.3. To justify these results, it is useful to observe that, for every $s \in \mathbb{N}, U_{s}\left(h_{s}\right) \cap U_{s}\left(\bar{h}_{s}\right)=U_{s}\left(\max \left\{h_{s}, \bar{h}_{s}\right\}\right)$.

Remark 4.6 If $a: \mathbb{R} \rightarrow \mathbb{R}$ is a periodic function such that the set $\Phi(a)$ is nonempty and closed and the set $\mathbb{R} \backslash \Phi(a)$ is nonempty and if, for every $s \in \mathbb{N}, h_{s}: \mathbb{R} \rightarrow \mathbb{R}$ is the function such that

$$
\forall t \in \mathbb{R}, \quad h_{s}(t)=a(s t),
$$

then the following assertions hold: (i) for every $s \in \mathbb{N}$, the set $\Phi\left(h_{s}\right)$ is nonempty and closed; (ii) for every $s \in \mathbb{N}$, the set $U_{s}\left(h_{s}\right)$ is nonempty; (iii) there is no function $h: \mathbb{R} \rightarrow \mathbb{R}$ such that the set $\Phi(h)$ has nonempty interior and condition ( $\left.*^{\prime \prime}\right)$ of Theorem 4.1 is satisfied; (iv) if $h: \mathbb{R} \rightarrow \mathbb{R}$ and condition ( $\left.*^{\prime \prime \prime}\right)$ of Theorem 4.1 is satisfied, then $\Phi(h)=\mathbb{R}$. It follows from assertions (iii) and (iv) that Theorems 4.1-4.3 cannot be applied to the study of the convergence of minimizers and minimum values of the functionals $F_{s}+G_{s}$ on the sets $U_{s}\left(h_{s}\right)$ with the functions $h_{s}$ defined by (4.19). The convergence of solutions of variational problems with implicit constraints defined by rapidly oscillating periodic functions was studied in our work [19], where we showed that, in the considered case, in contrast to the present paper, the corresponding limit problem is the minimization problem on $\mathbb{R}$ for a real function. In so doing, we introduced and used the notion of $\Gamma$-convergence of a sequence of functionals defined on variable Sobolev spaces to a function on the real line and did not assume that these spaces are strongly connected with a limit space.

\section{Comments and examples}

In this section, we give some comments and examples concerning the assumptions of Sect. 2, the conditions on the involved spaces and functionals, and the conditions of Theorems 4.14.3.

First, we discuss conditions $\left(*_{1}\right)-\left(*_{4}\right)$ stated at the beginning of Sect. 4.

As is known (see, for instance, [1]), condition $\left(*_{1}\right)$ is satisfied if the domain $\Omega$ is Lipschitz. In particular, bounded convex domains are Lipschitz domains. A more general assumption providing the fulfillment of condition $\left(*_{1}\right)$ is that $\Omega$ is an extension domain (see, for instance, [22, Chapter 1]). 
Condition $\left(*_{2}\right)$ is satisfied, in particular, if the domains $\Omega_{s}$ have a certain perforated structure. In this regard, see, for instance, [14, Sect. 2].

As far as condition $\left(*_{3}\right)$ is concerned, we note the following. In the case where the functions $\mu_{s}$ take a constant nonnegative value independent of $s$, theorems on the conditions for the $\Gamma$-convergence of the sequence of integral functionals $F_{S}$ with the integrands $f_{s}$ satisfying condition (2.1) follow from the results of $[12,13]$, where we studied the $\Gamma$-convergence of integral functionals defined on the spaces $W^{k, p}\left(\Omega_{s}\right)$ with an arbitrary $k \in \mathbb{N}$. In this case, the sequence $\left\{F_{S}\right\} \Gamma$-converges to an integral functional, in particular, if the domains $\Omega_{s}$ have a periodic structure and all the integrands $f_{s}$ coincide with the same integrand having a certain regularity (see [12]). Obviously, in the specified case, the sequence of norms $\left\|\mu_{s}\right\|_{L^{1}\left(\Omega_{s}\right)}$ is bounded and condition (*) of Theorem 4.1 is satisfied. In the more general case where $\mu_{s} \in L^{1}\left(\Omega_{s}\right)$ and $\mu_{s} \geqslant 0$ in $\Omega_{s}$ for every $s \in \mathbb{N}$ and, in addition, the inequality

$$
\limsup _{s \rightarrow \infty} \int_{Q \cap \Omega_{s}} \mu_{s} \mathrm{~d} x \leqslant \int_{Q \cap \Omega} \mu \mathrm{d} x
$$

holds for a nonnegative function $\mu \in L^{1}(\Omega)$ and every open cube $Q$ of $\mathbb{R}^{n}$, a theorem on the $\Gamma$-compactness of the sequence $\left\{F_{s}\right\}$ can be proved similarly to the corresponding result in [24]. It is easy to see that, in this case, the sequence of norms $\left\|\mu_{s}\right\|_{L^{1}\left(\Omega_{s}\right)}$ is bounded. Moreover, we note that there are examples of sequences of nonnegative functions $\mu_{s} \in$ $L^{1}\left(\Omega_{s}\right)$ where inequality (5.1) holds for a nonnegative function $\mu \in L^{1}(\Omega)$ and every open cube $Q$ of $\mathbb{R}^{n}$ and condition $(*)$ of Theorem 4.1 is satisfied but there is no function $\mu_{*}: \Omega \rightarrow \mathbb{R}$ such that, for every $s \in \mathbb{N}, \mu_{s} \leqslant \mu_{*}$ a.e. in $\Omega_{s}$. Such examples can be given using the functions constructed in [16].

Further, we consider an example where condition $\left(*_{4}\right)$ is satisfied.

Example 5.1 Let $b_{1}, b_{2}>0, \psi \in L^{1}(\Omega), \psi \geqslant 0$ in $\Omega$, and let $g: \Omega \times \mathbb{R} \rightarrow \mathbb{R}$ be a Carathéodory function such that, for almost every $x \in \Omega$ and every $\eta \in \mathbb{R}$,

$$
b_{1}|\eta|^{p}-\psi(x) \leqslant g(x, \eta) \leqslant b_{2}|\eta|^{p}+\psi(x) .
$$

It is easy to see that if $v \in W^{1, p}(\Omega)$, then $g(x, v) \in L^{1}(\Omega)$. Moreover, if $s \in \mathbb{N}$ and $v \in W^{1, p}\left(\Omega_{S}\right)$, then $g(x, v) \in L^{1}\left(\Omega_{S}\right)$.

Let $b_{3} \in\left(0, b_{1}\right), b_{4} \geqslant 0$, and let $J:[0,+\infty) \rightarrow \mathbb{R}$ be a continuous function such that

$$
\forall \eta \in[0,+\infty), \quad J(\eta) \geqslant-b_{3} \eta^{p}-b_{4} .
$$

Now, let, for every $s \in \mathbb{N}$, the functional $G_{s}$ be defined as follows:

$$
G_{S}(v)=\int_{\Omega_{S}} g(x, v) \mathrm{d} x+J\left(\|v\|_{L^{p}\left(\Omega_{s}\right)}\right), \quad v \in W^{1, p}\left(\Omega_{S}\right) .
$$

Using the properties of the functions $g$ and $J$, it is not difficult to verify that if conditions $\left(*_{1}\right)$ and $\left(*_{2}\right)$ are satisfied, then, for every $s \in \mathbb{N}$, the functional $G_{s}$ is weakly continuous on $W^{1, p}\left(\Omega_{s}\right)$. In addition, it follows from (5.2) and (5.3) that, for every $s \in \mathbb{N}$ and every $v \in W^{1, p}\left(\Omega_{s}\right)$, inequality (2.2) holds with $c_{3}=b_{1}-b_{3}$ and $c_{4}=b_{4}+\|\psi\|_{L^{1}(\Omega)}$. Using the properties of the functions $g$ and $J$, we also establish that the following assertion holds:

(A) for every function $v \in W^{1, p}(\Omega)$ and every sequence $v_{s} \in W^{1, p}\left(\Omega_{s}\right)$ with the property $\left\|v_{s}-q_{s} v\right\|_{L^{p}\left(\Omega_{s}\right)} \rightarrow 0$, we have $G_{s}\left(v_{s}\right)-G_{s}\left(q_{s} v\right) \rightarrow 0$.

Next, assume that the following condition is satisfied: 
(B) there exists a nonnegative bounded measurable function $b: \Omega \rightarrow \mathbb{R}$ such that, for every open cube $Q \subset \Omega$, we have meas $\left(Q \cap \Omega_{s}\right) \rightarrow \int_{Q} b \mathrm{~d} x$.

This condition implies that

$$
\forall v \in L^{1}(\Omega), \quad \int_{\Omega_{s}} v \mathrm{~d} x \rightarrow \int_{\Omega} b v \mathrm{~d} x .
$$

Now, let $G: W^{1, p}(\Omega) \rightarrow \mathbb{R}$ be the functional such that, for every function $v \in$ $W^{1, p}(\Omega)$,

$$
G(v)=\int_{\Omega} b g(x, v) \mathrm{d} x+J\left(\left\|b^{1 / p} v\right\|_{L^{p}(\Omega)}\right) .
$$

Using (5.4) and the continuity of the function $J$, we find that, for every function $v \in W^{1, p}(\Omega)$, $G_{s}\left(q_{s} v\right) \rightarrow G(v)$. This along with assertion (A) implies that for every function $v \in W^{1, p}(\Omega)$ and every sequence $v_{s} \in W^{1, p}\left(\Omega_{s}\right)$ with the property $\left\|v_{s}-q_{s} v\right\|_{L^{p}\left(\Omega_{s}\right)} \rightarrow 0$, we have $G_{s}\left(v_{s}\right) \rightarrow G(v)$. Thus, condition $\left(*_{4}\right)$ is satisfied.

We observe that, in the case where $\Omega$ is a Lipschitz domain and the domains $\Omega_{s}$ have a certain periodically perforated structure (see, for instance, [14, Sect. 2]), conditions $\left(*_{1}\right)$ and $\left(*_{2}\right)$ are satisfied along with condition $(\mathrm{B})$ in which the function $b$ takes a constant positive value.

We now discuss the conditions of Theorems 4.1-4.3 on functions $h_{s}$ and $h$. We begin with some general remarks.

First, we note that if $h: \mathbb{R} \rightarrow \mathbb{R}$ is a lower semicontinuous function on $\mathbb{R}$, then the set $\Phi(h)$ is closed. However, if $h: \mathbb{R} \rightarrow \mathbb{R}$ and the set $\Phi(h)$ is closed, then, in general, it is not true that the function $h$ is lower semicontinuous.

Next, we recall the notion of convergence of sets in the sense of Kuratowski [21, Sect. 29] applied to sets in $\mathbb{R}$. Let $\left\{\mathcal{B}_{s}\right\}$ be a sequence of sets in $\mathbb{R}$. We denote by Lim inf $\mathcal{B}_{s}$ the set of all numbers $t \in \mathbb{R}$ with the following property: if $\varepsilon>0$, then there exists $s^{(\varepsilon)} \in \mathbb{N}$ such that, for every $s \in \mathbb{N}, s \geqslant s^{(\varepsilon)}$, we have $(t-\varepsilon, t+\varepsilon) \bigcap \mathcal{B}_{s} \neq \varnothing$. We denote by Lim $\sup \mathcal{B}_{s}$ the set of all numbers $t \in \mathbb{R}$ such that, for every $\varepsilon>0$ and every $k \in \mathbb{N}$, we have $(t-\varepsilon, t+\varepsilon) \bigcap\left(\bigcup_{s=k}^{\infty} \mathcal{B}_{s}\right) \neq \varnothing$. We say that the sequence $\left\{\mathcal{B}_{s}\right\}$ converges to a set $\mathcal{B} \subset \mathbb{R}$ in the sense of Kuratowski if $\operatorname{Lim} \inf \mathcal{B}_{s}=\mathcal{B}$ and $\operatorname{Lim} \sup \mathcal{B}_{s}=\mathcal{B}$.

It is easy to see that if, for every $s \in \mathbb{N}, h_{s}: \mathbb{R} \rightarrow \mathbb{R}$ is a function such that the set $\Phi\left(h_{s}\right)$ is nonempty and if $h: \mathbb{R} \rightarrow \mathbb{R}$ is a function such that the set $\Phi(h)$ is nonempty, then: (i) conditions $\left(*^{\prime}\right)-\left(*^{\prime \prime \prime}\right)$ of Theorem 4.1 imply that the sequence $\left\{\Phi\left(h_{s}\right)\right\}$ converges to the set $\Phi(h)$ in the sense of Kuratowski; (ii) conditions $\left(*^{\prime}\right)$ and $\left(*^{\prime \prime \prime}\right)$ of Theorem 4.2 (or, equivalently, conditions $\left(*^{\prime}\right)$ and $\left(*^{\prime \prime}\right)$ of Theorem 4.3$)$ are satisfied if and only if the sequence $\left\{\Phi\left(h_{s}\right)\right\}$ converges to the set $\Phi(h)$ in the sense of Kuratowski.

However, in general, we cannot replace conditions $\left(*^{\prime}\right)-\left(*^{\prime \prime \prime}\right)$ of Theorem 4.1 by the requirement that the sequence $\left\{\Phi\left(h_{s}\right)\right\}$ converges to the set $\Phi(h)$ in the sense of Kuratowski without violating the conclusion of the theorem (see Example 5.10). Moreover, in general, we cannot remove condition $\left(*^{\prime \prime}\right)$ of Theorem 4.2 without violating the conclusion of this theorem (see Example 5.13).

Further, we note the following simple facts: (i) if $h: \mathbb{R} \rightarrow \mathbb{R}$ is a function such that the set $\Phi(h)$ is closed and has nonempty interior and if, for every $s \in \mathbb{N}$, we have $h_{s}=h$, then conditions $\left(*^{\prime}\right)-\left(*^{\prime \prime \prime}\right)$ of Theorem 4.2 are satisfied and, in particular, conditions $\left(*^{\prime \prime}\right)$ and $\left(*^{\prime \prime \prime}\right)$ of Theorem 4.1 are satisfied; (ii) if $h: \mathbb{R} \rightarrow \mathbb{R}$ is a function such that the set $\Phi(h)$ is nonempty and closed and if, for every $s \in \mathbb{N}$, we have $h_{s}=h$, then conditions $\left(*^{\prime}\right)$ and $\left(*^{\prime \prime}\right)$ of Theorem 4.3 are satisfied. 
A similar fact is described in the following proposition.

Proposition 5.2 Let $h: \mathbb{R} \rightarrow \mathbb{R}$ be a function such that the set $\Phi(h)$ is nonempty and closed, and assume that condition ( $\left.*^{\prime}\right)$ of Theorem 4.1 is satisfied. Let $\left\{\gamma_{s}\right\}$ be a sequence of functions in $C(\mathbb{R})$ converging to zero uniformly on every bounded closed interval of $\mathbb{R}$. Assume that, for every $s \in \mathbb{N}$, there exists $\eta_{s} \in \mathbb{R}$ such that $\eta_{s}-\gamma_{s}\left(\eta_{s}\right) \in \Phi(h)$. Let, for every $s \in \mathbb{N}$, $h_{s}: \mathbb{R} \rightarrow \mathbb{R}$ be the function defined as follows: $h_{s}(t)=h\left(t-\gamma_{s}(t)\right), t \in \mathbb{R}$. Then: (i) for every $s \in \mathbb{N}$, the set $\Phi\left(h_{s}\right)$ is nonempty and closed; (ii) conditions $\left(*^{\prime}\right)-\left(*^{\prime \prime \prime}\right)$ of Theorem 4.2 are satisfied.

Proof If $s \in \mathbb{N}$, then $\eta_{s} \in \Phi\left(h_{s}\right)$ and, in view of the closedness of the set $\Phi(h)$ and the continuity of the function $\gamma_{s}$, the set $\Phi\left(h_{s}\right)$ is closed. Thus, assertion (i) holds.

Next, let $t \in \Phi(h)$. Then, by condition $\left(*^{\prime}\right)$ of Theorem 4.1 , there exist $t_{1}, t_{2} \in \mathbb{R}$ such that $t_{1}<t_{2}$ and $t \in\left[t_{1}, t_{2}\right] \subset \Phi(h)$. First, suppose that $t \in\left(t_{1}, t_{2}\right)$. Since $\gamma_{s}(t) \rightarrow 0$, there exists $s^{\prime} \in \mathbb{N}$ such that, for every $s \in \mathbb{N}, s \geqslant s^{\prime}$, we have $\left|\gamma_{s}(t)\right| \leqslant \min \left\{t-t_{1}, t_{2}-t\right\}$. We define the sequence $\left\{r_{s}\right\}$ as follows: $r_{s}=\eta_{s}$ if $s \in \mathbb{N}, s \leqslant s^{\prime} ; r_{s}=t$ if $s \in \mathbb{N}, s>s^{\prime}$. It is easy to see that $r_{s} \rightarrow t$ and, for every $s \in \mathbb{N}, r_{s} \in \Phi\left(h_{s}\right)$. Now, let $t=t_{1}$. For every $s \in \mathbb{N}$, we define $\beta_{s}=\max \left\{\left|\gamma_{s}(\eta)\right|: \eta \in\left[t_{1}, t_{2}\right]\right\}$. Obviously, $\beta_{s} \rightarrow 0$. Then there exists $s^{\prime} \in \mathbb{N}$ such that, for every $s \in \mathbb{N}, s \geqslant s^{\prime}$, we have $\beta_{s} \leqslant\left(t_{2}-t_{1}\right) / 2$. We define the sequence $\left\{r_{s}\right\}$ as follows: $r_{s}=\eta_{s}$ if $s \in \mathbb{N}, s \leqslant s^{\prime} ; r_{s}=t+\beta_{s}$ if $s \in \mathbb{N}, s>s^{\prime}$. It is easy to see that $r_{s} \rightarrow t$ and, for every $s \in \mathbb{N}, r_{s} \in \Phi\left(h_{s}\right)$. The case $t=t_{2}$ is considered similarly. Thus, condition $\left(*^{\prime}\right)$ of Theorem 4.2 is satisfied.

We now assume that $t_{1}, t_{2} \in \mathbb{R}, t_{1}<t_{2},\left(t_{1}, t_{2}\right) \subset \Phi(h)$, and $0<\sigma<\left(t_{2}-t_{1}\right) / 2$. Since the sequence $\left\{\gamma_{s}\right\}$ converges to zero uniformly on $\left[t_{1}+\sigma, t_{2}-\sigma\right]$, there exists $\bar{s} \in \mathbb{N}$ such that, for every $s \in \mathbb{N}, s \geqslant \bar{s}$, and every $t \in\left[t_{1}+\sigma, t_{2}-\sigma\right]$, we have $\left|\gamma_{s}(t)\right|<\sigma$. Then $\left[t_{1}+\sigma, t_{2}-\sigma\right] \subset \Phi\left(h_{s}\right)$ if $s \in \mathbb{N}$ and $s \geqslant \bar{s}$. Thus, condition $\left(*^{\prime \prime}\right)$ of Theorem 4.2 is satisfied.

Finally, the closedness of the set $\Phi(h)$ and the specified uniform convergence of the sequence $\left\{\gamma_{s}\right\}$ to zero imply that condition $\left(*^{\prime \prime \prime}\right)$ of Theorem 4.2 is satisfied. Thus, assertion (ii) holds.

Example 5.3 If $h: \mathbb{R} \rightarrow \mathbb{R}$ is the function such that, for every $t \in \mathbb{R}, h(t)=\sin t$, and if, for every $s \in \mathbb{N}, \gamma_{s}: \mathbb{R} \rightarrow \mathbb{R}$ is the function defined by $\gamma_{s}(t)=t^{2} / s, t \in \mathbb{R}$, then the functions $h$ and $\gamma_{s}$ satisfy conditions of Proposition 5.2.

It is easy to see that if $h_{s}$ and $h$ are the functions described in the statement of Proposition 5.2 and $h \in C(\mathbb{R})$, then the sequence $\left\{h_{s}\right\}$ converges to the function $h$ uniformly on every bounded closed interval of $\mathbb{R}$.

However, we observe that, in general, the uniform convergence of a sequence of functions $h_{s}: \mathbb{R} \rightarrow \mathbb{R}$ to a function $h: \mathbb{R} \rightarrow \mathbb{R}$ on every bounded closed interval of $\mathbb{R}$ does not guarantee that the sequence $\left\{\Phi\left(h_{s}\right)\right\}$ converges to the set $\Phi(h)$ in the sense of Kuratowski. Therefore, the specified convergence of functions, in general, cannot provide that they satisfy all the conditions of each of Theorems 4.1-4.3.

For instance, if, for every $s \in \mathbb{N}, h_{s}: \mathbb{R} \rightarrow \mathbb{R}$ is the function defined by $h_{s}(t)=|t| / s$, $t \in \mathbb{R}$, and if $h: \mathbb{R} \rightarrow \mathbb{R}$ is the function defined by $h(t)=0, t \in \mathbb{R}$, then the sequence $\left\{h_{s}\right\}$ converges to the function $h$ uniformly on every bounded closed interval of $\mathbb{R}$. At the same time, $\Phi(h)=\mathbb{R}$ and, for every $s \in \mathbb{N}$, we have $\Phi\left(h_{s}\right)=\{0\}$. Hence, the sequence $\left\{\Phi\left(h_{s}\right)\right\}$ converges to the set $\{0\}$ in the sense of Kuratowski and, therefore, this sequence does not converge to the set $\Phi(h)$ in the same sense. As seen, in the case under consideration, condition $\left(*^{\prime \prime}\right)$ of Theorem 4.1 and conditions $\left(*^{\prime}\right)$ and $\left(*^{\prime \prime}\right)$ of Theorem 4.2 are not satisfied.

Let us state two propositions concerning some cases where the uniform convergence of a sequence of functions $h_{s}: \mathbb{R} \rightarrow \mathbb{R}$ to a function $h: \mathbb{R} \rightarrow \mathbb{R}$ on every bounded closed 
interval of $\mathbb{R}$ along with certain additional conditions implies the fulfillment of the conditions of Theorem 4.2 for these functions.

Proposition 5.4 Let, for every $s \in \mathbb{N}, h_{s}: \mathbb{R} \rightarrow \mathbb{R}$ be a function such that the set $\Phi\left(h_{s}\right)$ is nonempty. Let $h: \mathbb{R} \rightarrow \mathbb{R}$ be a lower semicontinuous function on $\mathbb{R}$ such that the set $\Phi(h)$ has nonempty interior. Assume that the sequence $\left\{h_{s}\right\}$ converges to the function $h$ uniformly on every bounded closed interval of $\mathbb{R}$. Moreover, assume that, for every $s \in \mathbb{N}, h_{s} \leqslant h$ in $\Phi(h)$. Then conditions $\left(*^{\prime}\right)-\left(*^{\prime \prime \prime}\right)$ of Theorem 4.2 are satisfied.

Proof We fix $t \in \Phi(h)$ and, for every $s \in \mathbb{N}$, we set $t_{s}=t$. If $s \in \mathbb{N}$, then we have $h_{s} \leqslant h$ in $\Phi(h)$ and, therefore, $h_{s}\left(t_{s}\right) \leqslant h(t)$. This along with the inclusion $t \in \Phi(h)$ implies that, for every $s \in \mathbb{N}, t_{s} \in \Phi\left(h_{s}\right)$. Thus, condition $\left(*^{\prime}\right)$ of Theorem 4.2 is satisfied. Condition $\left(*^{\prime \prime}\right)$ of Theorem 4.2 is also satisfied. This is a direct consequence of the fact that, for every $s \in \mathbb{N}$, $h_{s} \leqslant h$ in $\Phi(h)$.

Next, let $t_{s} \rightarrow t$ in $\mathbb{R}$, let $\left\{\tilde{s}_{j}\right\}$ be an increasing sequence in $\mathbb{N}$, and let, for every $j \in \mathbb{N}$, we have $t_{\tilde{s}_{j}} \in \Phi\left(h_{\tilde{s}_{j}}\right)$. It is clear that, for every $s \in \mathbb{N}$,

$$
h\left(t_{s}\right) \leqslant h_{s}\left(t_{s}\right)+\left|h_{s}\left(t_{s}\right)-h\left(t_{s}\right)\right| .
$$

Moreover, since $t_{s} \rightarrow t$ and the sequence $\left\{h_{s}\right\}$ converges to the function $h$ uniformly on $[t-1, t+1]$, we have $h_{s}\left(t_{s}\right)-h\left(t_{s}\right) \rightarrow 0$. This along with (5.5) and the inclusions $t_{\tilde{s}_{j}} \in \Phi\left(h_{\tilde{s}_{j}}\right)$, $j \in \mathbb{N}$, implies that $\liminf _{j \rightarrow \infty} h\left(t_{\tilde{s}_{j}}\right) \leqslant 0$. Hence, in view of the lower semicontinuity of the function $h$ and the fact that $t_{s} \rightarrow t$, we obtain the inequality $h(t) \leqslant 0$. Therefore, $t \in \Phi(h)$. Thus, condition $\left(*^{\prime \prime \prime}\right)$ of Theorem 4.2 is satisfied.

Proposition 5.5 Let, for every $s \in \mathbb{N}, h_{s}: \mathbb{R} \rightarrow \mathbb{R}$ be a function such that the set $\Phi\left(h_{s}\right)$ is nonempty. Let $h$ be a function in $C(\mathbb{R})$ such that the set $\Phi(h)$ has nonempty interior. Assume that the following conditions are satisfied:

$\left(\bar{*}^{\prime}\right)$ if $t \in \Phi(h)$, then there exists a sequence $\left\{\eta_{k}\right\} \subset \mathbb{R}$ such that $\eta_{k} \rightarrow t$ and, for every $k \in \mathbb{N}, h\left(\eta_{k}\right)<0$;

$\left(\bar{*}^{\prime \prime}\right)$ if $t_{1}, t_{2} \in \mathbb{R}, t_{1}<t_{2}$, and $\left(t_{1}, t_{2}\right) \subset \Phi(h)$, then $h<0$ in $\left(t_{1}, t_{2}\right)$.

Assume that the sequence $\left\{h_{s}\right\}$ converges to the function $h$ uniformly on every bounded closed interval of $\mathbb{R}$. Then conditions $\left(*^{\prime}\right)-\left(*^{\prime \prime \prime}\right)$ of Theorem 4.2 are satisfied.

Proof Let $t \in \Phi(h)$. Then, by condition $\left(\bar{*}^{\prime}\right)$, there exists a sequence $\left\{\eta_{k}\right\} \subset \mathbb{R}$ such that $\eta_{k} \rightarrow t$ and

$$
\forall k \in \mathbb{N}, \quad h\left(\eta_{k}\right)<0 .
$$

It is clear that, for every $k \in \mathbb{N}, h_{s}\left(\eta_{k}\right) \rightarrow h\left(\eta_{k}\right)$. This and (5.6) imply that there exists an increasing sequence $\left\{s_{k}\right\} \subset \mathbb{N}$ such that

$$
k \in \mathbb{N}, s \in \mathbb{N}, s \geqslant s_{k} \Longrightarrow h_{s}\left(\eta_{k}\right) \leqslant 0 .
$$

We fix a sequence $r_{s} \in \Phi\left(h_{s}\right)$ and define the sequence $\left\{t_{s}\right\}$ as follows: $t_{s}=r_{s}$ if $s \leqslant s_{1}$; $t_{s}=\eta_{k}$ if $s_{k}<s \leqslant s_{k+1}, k \in \mathbb{N}$. It is easy to see that $t_{s} \rightarrow t$. Moreover, using (5.7), we find that, for every $s \in \mathbb{N}, t_{s} \in \Phi\left(h_{s}\right)$. Thus, condition $\left(*^{\prime}\right)$ of Theorem 4.2 is satisfied.

Next, we assume that $t_{1}, t_{2} \in \mathbb{R}, t_{1}<t_{2},\left(t_{1}, t_{2}\right) \subset \Phi(h)$, and $0<\sigma<\left(t_{2}-t_{1}\right) / 2$. We define $\lambda_{*}=\max \left\{h(\eta): \eta \in\left[t_{1}+\sigma, t_{2}-\sigma\right]\right\}$. Using condition $\left(\bar{*}^{\prime \prime}\right)$, we establish that $\lambda_{*}<0$. Since the sequence $\left\{h_{s}\right\}$ converges to the function $h$ uniformly on $\left[t_{1}+\sigma, t_{2}-\sigma\right]$, there exists $\bar{s} \in \mathbb{N}$ such that, for every $s \in \mathbb{N}, s \geqslant \bar{s}$, and every $\eta \in\left[t_{1}+\sigma, t_{2}-\sigma\right]$, we have 
$\left|h_{s}(\eta)-h(\eta)\right| \leqslant-\lambda_{*}$. Then, for every $s \in \mathbb{N}, s \geqslant \bar{s}$, we have $\left[t_{1}+\sigma, t_{2}-\sigma\right] \subset \Phi\left(h_{s}\right)$. Thus, condition $\left(*^{\prime \prime}\right)$ of Theorem 4.2 is satisfied.

Finally, using the same arguments as in the corresponding place in the proof of Proposition 5.4, we establish that condition $\left(*^{\prime \prime \prime}\right)$ of Theorem 4.2 is satisfied.

Example 5.6 Let $h: \mathbb{R} \rightarrow \mathbb{R}$ be the function defined as follows:

$$
h(t)= \begin{cases}0 & \text { if } t=0, \\ 1 & \text { if } t \in[-1,1] \backslash\{0\}, \\ 2-t \operatorname{sign} t & \text { if } t \in(-\infty,-1) \cup(1,+\infty),\end{cases}
$$

and let, for every $s \in \mathbb{N}, h_{s}: \mathbb{R} \rightarrow \mathbb{R}$ be the function defined by

$$
h_{s}(t)=h(t)-\frac{1}{s}|t|\left(t^{2}-4\right), \quad t \in \mathbb{R} .
$$

It is easy to see that the functions $h_{s}$ and $h$ satisfy all the conditions of Proposition 5.4.

Example 5.7 Let $h: \mathbb{R} \rightarrow \mathbb{R}$ be the function defined as follows:

$$
h(t)= \begin{cases}0 & \text { if } t=0, \\ t \sin \frac{1}{t} & \text { if } t \neq 0,\end{cases}
$$

and let, for every $s \in \mathbb{N}, h_{s}: \mathbb{R} \rightarrow \mathbb{R}$ be the function defined by

$$
h_{s}(t)=h(t)+\frac{2^{t}}{s} \sin (s t), \quad t \in \mathbb{R} .
$$

It is not difficult to verify that the functions $h_{s}$ and $h$ satisfy all the conditions of Proposition 5.5.

Example 5.8 Let $\left\{\varphi_{s}\right\} \subset \mathbb{R}, \varphi \in \mathbb{R}$, and let $\varphi_{s} \rightarrow \varphi$. Let, for every $s \in \mathbb{N}, h_{s}: \mathbb{R} \rightarrow \mathbb{R}$ be the function defined by $h_{s}(t)=-t+\varphi_{s}, t \in \mathbb{R}$. Let $h: \mathbb{R} \rightarrow \mathbb{R}$ be the function such that, for every $t \in \mathbb{R}, h(t)=-t+\varphi$. For every $s \in \mathbb{N}$, we have $\Phi\left(h_{s}\right)=\left[\varphi_{s},+\infty\right)$. In addition, $\Phi(h)=[\varphi,+\infty)$. It is easy to verify that the functions $h_{s}$ and $h$ satisfy the corresponding conditions of Theorems 4.1 and 4.2 and, for every $s \in \mathbb{N}, U_{s}\left(h_{s}\right)=\left\{v \in W^{1, p}\left(\Omega_{s}\right): v \geqslant \varphi_{s}\right.$ a.e. in $\left.\Omega_{s}\right\}$. Similarly, let, for every $s \in \mathbb{N}, h_{s}: \mathbb{R} \rightarrow \mathbb{R}$ be the function defined by $h_{s}(t)=$ $t-\varphi_{s}, t \in \mathbb{R}$, and let $h: \mathbb{R} \rightarrow \mathbb{R}$ be the function such that, for every $t \in \mathbb{R}, h(t)=t-\varphi$. Then the functions $h_{s}$ and $h$ satisfy the corresponding conditions of Theorems 4.1 and 4.2 and, for every $s \in \mathbb{N}, U_{s}\left(h_{s}\right)=\left\{v \in W^{1, p}\left(\Omega_{s}\right): v \leqslant \varphi_{s}\right.$ a.e. in $\left.\Omega_{s}\right\}$. Finally, let, for every $s \in \mathbb{N}$, we have $\varphi_{s}, \psi_{s} \in \mathbb{R}$ and $\varphi_{s}<\psi_{s}$. Let $\varphi, \psi \in \mathbb{R}$ and $\varphi<\psi$. We assume that $\varphi_{s} \rightarrow \varphi$ and $\psi_{s} \rightarrow \psi$. Let, for every $s \in \mathbb{N}, h_{s}: \mathbb{R} \rightarrow \mathbb{R}$ be the function defined by $h_{s}(t)=\left(t-\varphi_{s}\right)\left(t-\psi_{s}\right), t \in \mathbb{R}$, and let $h: \mathbb{R} \rightarrow \mathbb{R}$ be the function such that, for every $t \in \mathbb{R}$, $h(t)=(t-\varphi)(t-\psi)$. Then the functions $h_{s}$ and $h$ satisfy the corresponding conditions of Theorems 4.1 and 4.2 and, for every $s \in \mathbb{N}, U_{s}\left(h_{s}\right)=\left\{v \in W^{1, p}\left(\Omega_{s}\right): \varphi_{s} \leqslant v \leqslant \psi_{s}\right.$ a.e. in $\Omega_{s}$ \}. Thus, Theorems 4.1 and 4.2 can be applied to variational problems with unilateral and bilateral constraints defined by real numbers.

Example 5.9 Let, for every $s \in \mathbb{N}, h_{s}: \mathbb{R} \rightarrow \mathbb{R}$ be the function defined by $h_{s}(t)=\cos t+$ $1-|t| / s, t \in \mathbb{R}$. Let $h: \mathbb{R} \rightarrow \mathbb{R}$ be the function such that, for every $t \in \mathbb{R}, h(t)=\cos t+1$. Obviously, for every $s \in \mathbb{N}$, the set $\Phi\left(h_{s}\right)$ is nonempty and closed. Moreover, $\Phi(h)=$ $\{(1+2 k) \pi: k \in \mathbb{Z}\}$. Hence, the set $\Phi(h)$ is nonempty and closed and has empty interior. It is easy to verify that the functions $h_{s}$ and $h$ satisfy conditions $\left(*^{\prime}\right)$ and $\left(*^{\prime \prime}\right)$ of Theorem 4.3. 
The next example justifies the importance of condition $\left(*^{\prime}\right)$ of Theorem 4.1 for the conclusion of this theorem. At the same time, this example shows that condition (4.11) is essential for the conclusion of Theorem 4.2.

Example 5.10 We assume that the domain $\Omega$ is Lipschitz and that, for every $s \in \mathbb{N}, \Omega_{s}=\Omega$. Then, obviously, conditions $\left(*_{1}\right)$ and $\left(*_{2}\right)$ stated at the beginning of Sect. 4 are satisfied.

It follows from [20, Example 4.16] that there exist numbers $\lambda_{1}, \lambda_{2}>0$ and a sequence $\left\{\tau_{s}\right\} \subset C_{0}^{\infty}(\Omega)$ such that

$$
\begin{aligned}
& \forall s \in \mathbb{N}, \quad\left|\nabla \tau_{s}\right| \leqslant \lambda_{1} \text { in } \Omega, \\
& \left\|\tau_{s}\right\|_{L^{p}(\Omega)} \rightarrow 0, \\
& \int_{\Omega}\left|\nabla \tau_{s}\right|^{p} \mathrm{~d} x \rightarrow \lambda_{2} .
\end{aligned}
$$

Assume that $c_{1}=2^{1-p}$ and $c_{2}=2^{p-1}$ and that, for every $s \in \mathbb{N}$ and every $x \in \Omega_{s}$, $\mu_{s}(x)=2^{p-1}\left|\nabla \tau_{s}(x)\right|^{p}$. Moreover, assume that, for every $s \in \mathbb{N}$ and every pair $(x, \xi) \in$ $\Omega_{s} \times \mathbb{R}^{n}$,

$$
f_{s}(x, \xi)=\left|\xi+\nabla \tau_{s}(x)\right|^{p} .
$$

In view of these assumptions and (5.8), the functions $\mu_{s}$ and $f_{s}$ satisfy the corresponding conditions of Sect. 2. In particular, if $s \in \mathbb{N}$, then, for every $x \in \Omega_{s}$ and every $\xi \in \mathbb{R}^{n}$, inequality (2.1) holds. Moreover, due to (5.8), condition (*) of Theorem 4.1 is satisfied. At the same time, by (5.10), we have $\left\|\mu_{s}\right\|_{L^{1}\left(\Omega_{s}\right)} \rightarrow 2^{p-1} \lambda_{2}$. This along with the inequality $\lambda_{2}>0$ implies that condition (4.11) is not satisfied.

By virtue of Definition 2.4 and (5.11), for every $s \in \mathbb{N}$ and every $v \in W^{1, p}\left(\Omega_{s}\right)$, we have

$$
F_{S}(v)=\int_{\Omega_{S}}\left|\nabla v+\nabla \tau_{s}\right|^{p} \mathrm{~d} x .
$$

Let $F: W^{1, p}(\Omega) \rightarrow \mathbb{R}$ be the functional such that, for every function $v \in W^{1, p}(\Omega)$,

$$
F(v)=\int_{\Omega}|\nabla v|^{p} \mathrm{~d} x
$$

Using the weak lower semicontinuity of the functional $F$ on $W^{1, p}(\Omega)$ along with (5.8), (5.9), (5.12), and (5.13), we establish that the sequence $\left\{F_{S}\right\} \Gamma$-converges to the functional $F$. Thus, condition $\left(*_{3}\right)$ stated at the beginning of Sect. 4 is satisfied.

Let, for every $s \in \mathbb{N}, u_{s}$ be the function in $W^{1, p}\left(\Omega_{s}\right)$ such that $u_{s}=-1 / s$ in $\Omega_{s}$. Assume that, for every $s \in \mathbb{N}$, the functional $G_{s}$ is defined by

$$
G_{s}(v)=\lambda_{1}^{p} \int_{\Omega_{s}}\left|v-u_{s}\right|^{p} \mathrm{~d} x, \quad v \in W^{1, p}\left(\Omega_{s}\right) .
$$

Let $G: W^{1, p}(\Omega) \rightarrow \mathbb{R}$ be the functional such that, for every function $v \in W^{1, p}(\Omega)$,

$$
G(v)=\lambda_{1}^{p} \int_{\Omega}|v|^{p} \mathrm{~d} x .
$$

Obviously, for every $s \in \mathbb{N}, G_{s}$ is a weakly continuous functional on $W^{1, p}\left(\Omega_{s}\right)$. In addition, for every $s \in \mathbb{N}$ and every $v \in W^{1, p}\left(\Omega_{s}\right)$, inequality (2.2) holds with $c_{3}=\left(\lambda_{1} / 2\right)^{p}$ and $c_{4}=\lambda_{1}^{p}$ meas $\Omega$. Furthermore, for every function $v \in W^{1, p}(\Omega)$ and every sequence $v_{s} \in$ $W^{1, p}\left(\Omega_{s}\right)$ with the property $\left\|v_{s}-q_{s} v\right\|_{L^{p}\left(\Omega_{s}\right)} \rightarrow 0$, we have $G_{s}\left(v_{s}\right) \rightarrow G(v)$. Thus, condition $\left(*_{4}\right)$ stated at the beginning of Sect. 4 is satisfied. 
Next, let $h: \mathbb{R} \rightarrow \mathbb{R}$ be the function defined as follows:

$$
h(t)= \begin{cases}|t| & \text { if } t \in(-\infty, 1), \\ -t+2 & \text { if } t \in[1,3], \\ t-4 & \text { if } t \in(3,+\infty) .\end{cases}
$$

We have $\Phi(h)=\{0\} \cup[2,4]$. Hence, the set $\Phi(h)$ is closed and has nonempty interior. At the same time, condition $\left(*^{\prime}\right)$ of Theorem 4.1 is not satisfied.

Now, let, for every $s \in \mathbb{N}, h_{s}: \mathbb{R} \rightarrow \mathbb{R}$ be the function defined by

$$
h_{s}(t)=h\left(t+\frac{1}{s}\right), \quad t \in \mathbb{R} .
$$

For every $s \in \mathbb{N}$, we have

$$
\Phi\left(h_{s}\right)=\{-1 / s\} \cup[2-1 / s, 4-1 / s] .
$$

Hence, for every $s \in \mathbb{N}$, the set $\Phi\left(h_{s}\right)$ is nonempty and closed. It is easy to verify that the sequence $\left\{\Phi\left(h_{s}\right)\right\}$ converges to the set $\Phi(h)$ in the sense of Kuratowski and conditions $\left(*^{\prime \prime}\right)$ and $\left(*^{\prime \prime \prime}\right)$ of Theorem 4.1 (or, equivalently, conditions $\left(*^{\prime \prime}\right)$ and $\left(*^{\prime \prime \prime}\right)$ of Theorem 4.2) are satisfied. In addition, condition $\left(*^{\prime}\right)$ of Theorem 4.2 is satisfied.

Next, we fix $s \in \mathbb{N}$. Obviously, $u_{s} \in U_{s}\left(h_{s}\right)$. Fixing an arbitrary $v \in U_{s}\left(h_{s}\right)$, we estimate $\left(F_{s}+G_{s}\right)(v)$ from below. Assume that $\underline{m}(v)<\bar{m}(v)$. Then, by virtue of Proposition 3.3, we have $(\underline{m}(v), \bar{m}(v)) \subset \Phi\left(h_{s}\right)$. This and (5.16) imply that $(\underline{m}(v), \bar{m}(v)) \subset[2-1 / s, 4-1 / s]$. Therefore, $v-u_{s} \geqslant 2$ a.e. in $\Omega_{s}$. Using this fact along with (5.12), (5.14), and (5.8), we obtain

$$
\begin{aligned}
\left(F_{s}+G_{s}\right)(v) & \geqslant \lambda_{1}^{p} \int_{\Omega_{s}}\left|v-u_{s}\right|^{p} \mathrm{~d} x>\lambda_{1}^{p} \text { meas } \Omega \\
& \geqslant \int_{\Omega_{s}}\left|\nabla \tau_{s}\right|^{p} \mathrm{~d} x=\left(F_{s}+G_{s}\right)\left(u_{s}\right) .
\end{aligned}
$$

Now, let $\underline{m}(v)=\bar{m}(v)$. Then $\underline{m}(v) \in \mathbb{R}$ and $v=\underline{m}(v)$ a.e. in $\Omega_{s}$. This along with the inclusion $\bar{v} \in U_{s}\left(h_{s}\right)$ yields the inclusion $\underline{m}(v) \in \Phi\left(h_{s}\right)$. Therefore, in view of (5.16), we have either $\underline{m}(v)=-1 / s$ or $\underline{m}(v) \in[2-1 / s, 4-1 / s]$. In the first case, $v=u_{s}$ a.e. in $\Omega_{s}$. Hence, $\left(F_{s}+G_{s}\right)(v)=\left(F_{s}+G_{s}\right)\left(u_{s}\right)$. In the second case, $v-u_{s} \geqslant 2$ a.e. in $\Omega_{s}$ and, similarly to (5.17), we find that $\left(F_{s}+G_{s}\right)(v)>\left(F_{s}+G_{s}\right)\left(u_{s}\right)$.

In view of the above considerations, for every $s \in \mathbb{N}$, the function $u_{s}$ belongs to the set $U_{s}\left(h_{s}\right)$ and minimizes the functional $F_{s}+G_{s}$ on the set $U_{s}\left(h_{s}\right)$.

Thus, all the conditions of Theorem 4.1 are satisfied except for condition $\left(*^{\prime}\right)$. Moreover, all the conditions of Theorem 4.2 are satisfied except for condition (4.11). At the same time, the conclusions of these theorems do not hold for the sequence $\left\{u_{s}\right\}$.

Indeed, suppose that the conclusion of Theorem 4.1 holds for the sequence $\left\{u_{s}\right\}$. Then there exist an increasing sequence $\left\{s_{j}\right\} \subset \mathbb{N}$ and a function $u \in W^{1, p}(\Omega)$ such that

$$
\begin{aligned}
\left\|u_{s_{j}}-q_{s_{j}} u\right\|_{L^{p}\left(\Omega_{s_{j}}\right)} & \rightarrow 0, \\
\left(F_{s_{j}}+G_{s_{j}}\right)\left(u_{s_{j}}\right) & \rightarrow(F+G)(u) .
\end{aligned}
$$

Since, for every $s \in \mathbb{N}$, we have $u_{s}=-1 / s$ in $\Omega_{s}$ and $\Omega_{s}=\Omega$, using (5.18), we find that $u=0$ a.e. in $\Omega$. Then, by (5.13) and (5.15), we have $(F+G)(u)=0$. This along with (5.19) implies that $\left(F_{s_{j}}+G_{s_{j}}\right)\left(u_{s_{j}}\right) \rightarrow 0$. However, in view of (5.10) and (5.17), we have $\left(F_{s_{j}}+G_{s_{j}}\right)\left(u_{s_{j}}\right) \rightarrow \lambda_{2} \neq 0$. The obtained contradiction proves that the conclusion of 
Theorem 4.1 does not hold for the sequence $\left\{u_{s}\right\}$. Similarly, the conclusion of Theorem 4.2 does not hold for the sequence $\left\{u_{s}\right\}$.

The next example justifies the importance of condition $\left(*^{\prime}\right)$ of Theorem 4.2 for the conclusion of this theorem.

Example 5.11 Assume that the domain $\Omega$ is Lipschitz and that, for every $s \in \mathbb{N}, \Omega_{s}=\Omega$. Then, obviously, conditions $\left(*_{1}\right)$ and $\left(*_{2}\right)$ stated at the beginning of Sect. 4 are satisfied.

Assume that $c_{1}=c_{2}=1$ and that, for every $s \in \mathbb{N}, \mu_{s}=0$ in $\Omega_{s}$. Moreover, assume that, for every $s \in \mathbb{N}$ and every pair $(x, \xi) \in \Omega_{s} \times \mathbb{R}^{n}$,

$$
f_{s}(x, \xi)=|\xi|^{p} \text {. }
$$

In view of these assumptions, the functions $\mu_{s}$ and $f_{s}$ satisfy the corresponding conditions of Sect. 2. In particular, if $s \in \mathbb{N}$, then, for every $x \in \Omega_{s}$ and every $\xi \in \mathbb{R}^{n}$, inequality (2.1) holds. Moreover, condition (4.11) is satisfied.

Let $F, G: W^{1, p}(\Omega) \rightarrow \mathbb{R}$ be the functionals such that, for every function $v \in W^{1, p}(\Omega)$,

$$
F(v)=\int_{\Omega}|\nabla v|^{p} \mathrm{~d} x, \quad G(v)=\int_{\Omega}|v|^{p} \mathrm{~d} x .
$$

By virtue of Definition 2.4 and (5.20), for every $s \in \mathbb{N}$ and every $v \in W^{1, p}\left(\Omega_{S}\right)$, we have $F_{s}(v)=F(v)$. It is clear that the sequence $\left\{F_{s}\right\} \Gamma$-converges to the functional $F$. Thus, condition $\left(*_{3}\right)$ stated at the beginning of Sect. 4 is satisfied.

Assume that, for every $s \in \mathbb{N}, G_{s}=G$. Obviously, for every $s \in \mathbb{N}, G_{s}$ is a weakly continuous functional on $W^{1, p}\left(\Omega_{s}\right)$. In addition, for every $s \in \mathbb{N}$ and every $v \in W^{1, p}\left(\Omega_{s}\right)$, inequality (2.2) holds with $c_{3}=1$ and $c_{4}=0$. Furthermore, for every function $v \in W^{1, p}(\Omega)$ and every sequence $v_{s} \in W^{1, p}\left(\Omega_{S}\right)$ with the property $\left\|v_{s}-q_{s} v\right\|_{L^{p}\left(\Omega_{s}\right)} \rightarrow 0$, we have $G_{s}\left(v_{s}\right) \rightarrow G(v)$. Thus, condition (*4) stated at the beginning of Sect. 4 is satisfied.

Let, for every $s \in \mathbb{N}, h_{s}: \mathbb{R} \rightarrow \mathbb{R}$ be the function defined as follows:

$$
h_{s}(t)= \begin{cases}|t|+1 / s & \text { if } t \leqslant 1-1 / s \\ -t+2-1 / s & \text { if } t>1-1 / s .\end{cases}
$$

For every $s \in \mathbb{N}$, we have

$$
\Phi\left(h_{s}\right)=[2-1 / s,+\infty) .
$$

Hence, for every $s \in \mathbb{N}$, the set $\Phi\left(h_{s}\right)$ is nonempty and closed.

Let $h: \mathbb{R} \rightarrow \mathbb{R}$ be the function defined as follows:

$$
h(t)= \begin{cases}|t| & \text { if } t \leqslant 1, \\ -t+2 & \text { if } t>1 .\end{cases}
$$

We have

$$
\Phi(h)=\{0\} \cup[2,+\infty) .
$$

Hence, the set $\Phi(h)$ is closed and has nonempty interior.

Using (5.21) and (5.22), we verify that condition $\left(*^{\prime}\right)$ of Theorem 4.2 is not satisfied and conditions $\left(*^{\prime \prime}\right)$ and $\left(*^{\prime \prime \prime}\right)$ of Theorem 4.2 are satisfied.

It is clear that, for every $s \in \mathbb{N}$, there exists a unique function $u_{s} \in U_{s}\left(h_{s}\right)$ minimizing the functional $F_{s}+G_{s}$ on the set $U_{s}\left(h_{s}\right)$.

As we now see, all the conditions of Theorem 4.2 are satisfied except for condition $\left(*^{\prime}\right)$. At the same time, the conclusion of Theorem 4.2 does not hold for the sequence $\left\{u_{s}\right\}$. 
Indeed, suppose that the conclusion of Theorem 4.2 holds for the sequence $\left\{u_{s}\right\}$. Then there exist an increasing sequence $\left\{s_{j}\right\} \subset \mathbb{N}$ and a function $u \in U(h)$ such that the function $u$ minimizes the functional $F+G$ on the set $U(h)$ and

$$
\left\|u_{s_{j}}-q_{s_{j}} u\right\|_{L^{p}\left(\Omega_{s_{j}}\right)} \rightarrow 0 .
$$

Let $\theta: \Omega \rightarrow \mathbb{R}$ be the function such that, for every $x \in \Omega, \theta(x)=0$. Obviously, $\theta \in U(h)$. Then $(F+G)(u) \leqslant(F+G)(\theta)$. Hence, taking into account the definitions of the functionals $F$ and $G$, we derive that $u=\theta$ a.e. in $\Omega$. This and (5.23) imply that

$$
\left\|u_{s_{j}}\right\|_{L^{p}\left(\Omega_{s_{j}}\right)} \rightarrow 0 .
$$

On the other hand, using the inclusions $u_{s} \in U_{s}\left(h_{s}\right), s \in \mathbb{N}$, we find that, for every $s \in \mathbb{N}$, $u_{s} \geqslant 1$ a.e. in $\Omega_{s}$. Consequently, for every $s \in \mathbb{N},\left\|u_{s}\right\|_{L^{p}\left(\Omega_{s}\right)} \geqslant(\text { meas } \Omega)^{1 / p}$, which contradicts (5.24). The obtained contradiction proves that the conclusion of Theorem 4.2 does not hold for the sequence $\left\{u_{s}\right\}$.

Finally, we observe that the sequence $\left\{\Phi\left(h_{s}\right)\right\}$ does not converge to the set $\Phi(h)$ in the sense of Kuratowski.

Remark 5.12 An example justifying the importance of condition ( $\left.*^{\prime}\right)$ of Theorem 4.3 for the conclusion of this theorem is easily obtained from Example 5.11 if we replace the functions $h_{s}$ and $h$ defined in Example 5.11 by the functions $h_{s}: \mathbb{R} \rightarrow \mathbb{R}$ and $h: \mathbb{R} \rightarrow \mathbb{R}$ defined as follows:

$$
h_{s}(t)=\left\{\begin{array}{ll}
|t|+1 / s & \text { if } t \leqslant 1-1 / s, \\
|t-2+1 / s| & \text { if } t>1-1 / s,
\end{array} \quad h(t)= \begin{cases}|t| & \text { if } t \leqslant 1, \\
|t-2| & \text { if } t>1 .\end{cases}\right.
$$

The next example justifies the importance of condition $\left(*^{\prime \prime}\right)$ of Theorem 4.1 for the conclusion of this theorem. At the same time, this example shows that the same condition is essential for the conclusion of Theorem 4.2.

Example 5.13 Assume that the domains $\Omega$ and $\Omega_{s}$, the constants $c_{1}$ and $c_{2}$, and the functions $\mu_{s}$ and $f_{s}$ are the same as in Example 5.11. In addition, consider the same functional $F$ as in the mentioned example. Then conditions $\left(*_{1}\right)-\left(*_{3}\right)$ stated at the beginning of Sect. 4 are satisfied. Besides that, condition (*) of Theorem 4.1 and condition (4.11) are satisfied.

Next, we fix a function $\varphi \in W^{1, p}(\Omega)$ such that $\underline{m}(\varphi) \neq \bar{m}(\varphi)$, and let $b: \mathbb{R} \rightarrow \mathbb{R}$ be the function such that, for every $t \in \mathbb{R}$,

$$
b(t)=\int_{\Omega}|t-\varphi|^{p} \mathrm{~d} x .
$$

It is clear that $b \in C(\mathbb{R})$. Moreover, since $\underline{m}(\varphi) \neq \bar{m}(\varphi)$, for every $t \in \mathbb{R}$, we have $b(t)>0$.

We define

$$
t_{*}=2\left\{\frac{1}{\text { meas } \Omega} \int_{\Omega}\left(|\varphi|^{p}+|\nabla \varphi|^{p}\right) \mathrm{d} x\right\}^{1 / p} .
$$

Obviously, $t_{*}>0$. We set $M=\min \left\{b(t): t \in\left[-t_{*}, t_{*}\right]\right\}$. It is clear that $M>0$. We fix $\lambda \in \mathbb{R}$ such that

$$
\lambda>1+\frac{1}{M} \int_{\Omega}|\nabla \varphi|^{p} \mathrm{~d} x
$$

It is easy to verify that

$$
\forall t \in \mathbb{R}, \quad \lambda b(t)>\int_{\Omega}|\nabla \varphi|^{p} \mathrm{~d} x .
$$


Now, let $G: W^{1, p}(\Omega) \rightarrow \mathbb{R}$ be the functional such that, for every function $v \in W^{1, p}(\Omega)$,

$$
G(v)=\lambda \int_{\Omega}|v-\varphi|^{p} \mathrm{~d} x .
$$

Assume that, for every $s \in \mathbb{N}, G_{s}=G$. Obviously, for every $s \in \mathbb{N}, G_{s}$ is a weakly continuous functional on $W^{1, p}\left(\Omega_{s}\right)$. In addition, for every $s \in \mathbb{N}$ and every $v \in W^{1, p}\left(\Omega_{S}\right)$, inequality (2.2) holds with $c_{3}=2^{-p} \lambda$ and $c_{4}=\lambda\|\varphi\|_{L^{p}(\Omega)}^{p}$. Furthermore, for every function $v \in W^{1, p}(\Omega)$ and every sequence $v_{s} \in W^{1, p}\left(\Omega_{s}\right)$ with the property $\left\|v_{s}-q_{s} v\right\|_{L^{p}\left(\Omega_{s}\right)} \rightarrow 0$, we have $G_{s}\left(v_{s}\right) \rightarrow G(v)$. Thus, condition $\left(*_{4}\right)$ stated at the beginning of Sect. 4 is satisfied.

Let, for every $s \in \mathbb{N}, h_{s}: \mathbb{R} \rightarrow \mathbb{R}$ be the function defined as follows:

$$
h_{s}(t)=\sin (s t), t \in \mathbb{R} .
$$

It is clear that, for every $s \in \mathbb{N}$, the set $\Phi\left(h_{s}\right)$ is nonempty and closed.

Let $h: \mathbb{R} \rightarrow \mathbb{R}$ be the function such that, for every $t \in \mathbb{R}, h(t)=0$. We have $\Phi(h)=\mathbb{R}$. Hence, the set $\Phi(h)$ is closed and has nonempty interior.

It is easy to verify that conditions $\left(*^{\prime}\right)$ and $\left(*^{\prime \prime \prime}\right)$ of Theorem 4.1 and conditions $\left(*^{\prime}\right)$ and $\left(*^{\prime \prime \prime}\right)$ of Theorem 4.2 are satisfied but condition $\left(*^{\prime \prime}\right)$ of Theorem 4.1 (i.e., condition $\left(*^{\prime \prime}\right)$ of Theorem 4.2) is not satisfied.

Let, for every $s \in \mathbb{N}, u_{s}$ be a function in $U_{s}\left(h_{s}\right)$ minimizing the functional $F_{s}+G_{s}$ on the set $U_{s}\left(h_{s}\right)$.

As we now see, all the conditions of Theorem 4.1 are satisfied except for condition $\left(*^{\prime \prime}\right)$. The same relates to the conditions of Theorem 4.2. However, the conclusions of these theorems do not hold for the sequence $\left\{u_{s}\right\}$.

Indeed, suppose that the conclusion of Theorem 4.1 holds for the sequence $\left\{u_{s}\right\}$. Then there exist an increasing sequence $\left\{\bar{s}_{k}\right\} \subset \mathbb{N}$ and a function $u \in U(h)$ such that the function $u$ minimizes the functional $F+G$ on the set $U(h)$ and

$$
\left\|u_{\bar{s}_{k}}-q_{\bar{s}_{k}} u\right\|_{L^{p}\left(\Omega_{\bar{s}_{k}}\right)} \rightarrow 0 .
$$

It is easy to verify that the sequence of norms $\left\|u_{s}\right\|_{W^{1, p}\left(\Omega_{s}\right)}$ is bounded. Then, by Proposition 8 in [19], there exist an increasing sequence $\left\{s_{j}\right\} \subset\left\{\bar{s}_{k}\right\}$ and a number $t_{0} \in \mathbb{R}$ such that $\left\|u_{s_{j}}-t_{0}\right\|_{L^{p}\left(\Omega_{s_{j}}\right)} \rightarrow 0$. The latter limit relation along with (5.26) implies that $u=t_{0}$ a.e. in $\Omega$. Then we obtain

$$
(F+G)(u)=G(u)=\lambda \int_{\Omega}\left|t_{0}-\varphi\right|^{p} \mathrm{~d} x=\lambda b\left(t_{0}\right) .
$$

On the other hand, since $\varphi \in W^{1, p}(\Omega), U(h)=W^{1, p}(\Omega)$, and the function $u$ minimizes the functional $F+G$ on the set $U(h)$, we obtain

$$
(F+G)(u) \leqslant(F+G)(\varphi)=F(\varphi)=\int_{\Omega}|\nabla \varphi|^{p} \mathrm{~d} x .
$$

This along with (5.27) yields the inequality

$$
\lambda b\left(t_{0}\right) \leqslant \int_{\Omega}|\nabla \varphi|^{p} \mathrm{~d} x .
$$

However, this inequality contradicts (5.25). The obtained contradiction proves that the conclusion of Theorem 4.1 does not hold for the sequence $\left\{u_{s}\right\}$. Similarly, the conclusion of Theorem 4.2 does not hold for the sequence $\left\{u_{s}\right\}$.

Finally, we note that, in the given example, the sequence $\left\{\Phi\left(h_{S}\right)\right\}$ converges to the set $\Phi(h)$ in the sense of Kuratowski. 
Remark 5.14 If we have a sequence of functions $v_{s} \in W^{1, p}\left(\Omega_{S}\right)$, a function $v \in W^{1, p}(\Omega)$, and an increasing sequence $\left\{s_{j}\right\} \subset \mathbb{N}$ and if $\left\|v_{s_{j}}-q_{s_{j}} v\right\|_{L^{p}\left(\Omega_{s_{j}}\right)} \rightarrow 0$, we say that the sequence $\left\{v_{s_{j}}\right\}$ weakly converges to $v$. It is easy to see that, for given functions $h_{s}, h: \mathbb{R} \rightarrow$ $\mathbb{R}$, condition $\left(*^{\prime \prime \prime}\right)$ of Theorem 4.1 is necessary for the limit of every weakly convergent subsequence of any sequence $v_{s} \in U_{s}\left(h_{s}\right)$ to belong to the set $U(h)$.

At last, we give an example to justify the importance of condition (4.11) for the conclusion of Theorem 4.3.

Example 5.15 Assume that the domains $\Omega$ and $\Omega_{s}$, the constants $c_{1}$ and $c_{2}$, and the functions $\mu_{s}$ and $f_{s}$ are the same as in Example 5.10. Then conditions $\left(*_{1}\right)$ and $\left(*_{2}\right)$ stated at the beginning of Sect. 4 are satisfied. At the same time, condition (4.11) is not satisfied.

Let, for every $s \in \mathbb{N}, u_{s}$ be the function in $W^{1, p}\left(\Omega_{s}\right)$ such that $u_{s}=1 / s$ in $\Omega_{s}$. Assume that, for every $s \in \mathbb{N}$, the functional $G_{s}$ is defined by

$$
G_{S}(v)=\int_{\Omega_{s}}\left|v-u_{s}\right|^{p} \mathrm{~d} x, \quad v \in W^{1, p}\left(\Omega_{s}\right) .
$$

Let $G: W^{1, p}(\Omega) \rightarrow \mathbb{R}$ be the functional such that, for every function $v \in W^{1, p}(\Omega)$,

$$
G(v)=\int_{\Omega}|v|^{p} \mathrm{~d} x
$$

Obviously, for every $s \in \mathbb{N}, G_{s}$ is a weakly continuous functional on $W^{1, p}\left(\Omega_{s}\right)$. In addition, for every $s \in \mathbb{N}$ and every $v \in W^{1, p}\left(\Omega_{s}\right)$, inequality (2.2) holds with $c_{3}=2^{-p}$ and $c_{4}=$ meas $\Omega$. Furthermore, for every function $v \in W^{1, p}(\Omega)$ and every sequence $v_{s} \in W^{1, p}\left(\Omega_{s}\right)$ with the property $\left\|v_{s}-q_{s} v\right\|_{L^{p}\left(\Omega_{s}\right)} \rightarrow 0$, we have $G_{s}\left(v_{s}\right) \rightarrow G(v)$. Thus, condition $\left(*_{4}\right)$ stated at the beginning of Sect. 4 is satisfied.

Next, let, for every $s \in \mathbb{N}, h_{s}: \mathbb{R} \rightarrow \mathbb{R}$ be the function defined by

$$
h_{s}(t)=|t-1 / s|, \quad t \in \mathbb{R} .
$$

For every $s \in \mathbb{N}$, we have $\Phi\left(h_{s}\right)=\{1 / s\}$. Hence, for every $s \in \mathbb{N}$, the set $\Phi\left(h_{s}\right)$ is nonempty and closed. Let $h: \mathbb{R} \rightarrow \mathbb{R}$ be the function such that, for every $t \in \mathbb{R}, h(t)=|t|$. We have $\Phi(h)=\{0\}$. Hence, the set $\Phi(h)$ is nonempty and closed and has empty interior. It is easy to see that conditions $\left(*^{\prime}\right)$ and $\left(*^{\prime \prime}\right)$ of Theorem 4.3 are satisfied.

Obviously, for every $s \in \mathbb{N}$, the function $u_{s}$ belongs to the set $U_{s}\left(h_{s}\right)$ and minimizes the functional $F_{s}+G_{s}$ on this set.

Thus, all the conditions of Theorem 4.3 are satisfied except for condition (4.11). At the same time, the conclusion of this theorem does not hold for the sequence $\left\{u_{s}\right\}$.

Indeed, suppose that the conclusion of Theorem 4.3 holds for the sequence $\left\{u_{s}\right\}$. Then there exist an increasing sequence $\left\{s_{j}\right\} \subset \mathbb{N}$ and a function $u \in U(h)$ such that $\left(F_{s_{j}}+G_{s_{j}}\right)\left(u_{s_{j}}\right) \rightarrow$ $G(u)$. The inclusion $u \in U(h)$ implies that $u=0$ a.e. in $\Omega$. Then $G(u)=0$, and we have $\left(F_{s_{j}}+G_{s_{j}}\right)\left(u_{s_{j}}\right) \rightarrow 0$. However, in view of (5.10) and (5.12), we have $\left(F_{s_{j}}+G_{s_{j}}\right)\left(u_{s_{j}}\right) \rightarrow$ $\lambda_{2} \neq 0$. The obtained contradiction proves that the conclusion of Theorem 4.3 does not hold for the sequence $\left\{u_{s}\right\}$.

Acknowledgements This work was partially supported by the Russian Academic Excellence Project (agreement no. 02.A03.21.0006 of August 27, 2013, between the Ministry of Education and Science of the Russian Federation and Ural Federal University). 


\section{References}

1. Adams, R.A.: Sobolev Spaces. Academic Press, New York (1975)

2. Boccardo, L., Murat, F.: Homogenization of nonlinear unilateral problems. In: Dal Maso, G., Dell'Antonio, G.F. (eds.) Composite Media and Homogenization Theory, Progress in Nonlinear Differential Equations Applications 5, pp. 81-105. Birkhäuser, Boston (1991)

3. Dal Maso, G.: Asymptotic behaviour of minimum problems with bilateral obstacles. Ann. Mat. Pura Appl. (4) 129(1), 327-366 (1981)

4. Dal Maso, G.: Limits of minimum problems for general integral functionals with unilateral obstacles. Atti Accad. Naz. Lincei. Rend. Cl. Sci. Fis. Mat. Natur. (8) 74(2), 55-61 (1983)

5. Dal Maso, G.: An Introduction to $\Gamma$-Convergence. Birkhäuser, Boston (1993)

6. Dal Maso, G., Defranceschi, A.: Convergence of unilateral problems for monotone operators. J. Anal. Math. 53(1), 269-289 (1989)

7. Defranceschi, A., Vitali, E.: Limits of minimum problems with convex obstacles for vector valued functions. Appl. Anal. 52(1-4), 1-33 (1994)

8. De Giorgi, E., Franzoni, T.: Su un tipo di convergenza variazionale. Atti Accad. Naz. Lincei. Rend. Cl. Sci. Fis. Mat. Natur. (8) 58(6), 842-850 (1975)

9. Evans, L.C.: Partial Differential Equations. AMS, Providence (1998)

10. Khruslov, E.Ya.: The asymptotic behavior of solutions of the second boundary value problem under fragmentation of the boundary of the domain. Math. USSR-Sb. 35(2), 266-282 (1979)

11. Kovalevskii, A.A.: Some problems connected with the problem of averaging variational problems for functionals with a variable domain. In: Mitropol'skii, Yu.A. (ed.) Current Analysis and its Applications, pp. 62-70. Naukova Dumka, Kiev (1989) [in Russian]

12. Kovalevskij, A.A.: Conditions of the $\Gamma$-convergence and homogenization of integral functionals with different domains of the definition. Dokl. Akad. Nauk Ukr. SSR No. 4, 5-8 (1991). [in Russian]

13. Kovalevskii, A.A.: On necessary and sufficient conditions for the $\Gamma$-convergence of integral functionals with different domains of definition. Nelinein. Granichnye Zadachi 4, 29-39 (1992). [in Russian]

14. Kovalevskii, A.A.: $G$-convergence and homogenization of nonlinear elliptic operators in divergence form with variable domain. Russ. Acad. Sci. Izv. Math. 44(3), 431-460 (1995)

15. Kovalevsky, A.A.: Obstacle problems in variable domains. Complex Var. Elliptic Equ. 56(12), 1071-1083 (2011)

16. Kovalevsky, A.A.: On $L^{1}$-functions with a very singular behaviour. Nonlinear Anal. 85, 66-77 (2013)

17. Kovalevsky, A.A.: On the convergence of solutions of variational problems with bilateral obstacles in variable domains. Proc. Steklov Inst. Math. 296(Suppl. 1), 151-163 (2017)

18. Kovalevsky, A.A.: On the convergence of solutions of variational problems with implicit pointwise constraints in variable domains. Funct. Anal. Appl. 52(2), 147-150 (2018)

19. Kovalevsky, A.A.: On the convergence of solutions of variational problems with implicit constraints defined by rapidly oscillating functions. Trudy Inst. Mat. Mekh. UrO RAN 24(2), 107-122 (2018). [in Russian]

20. Kovalevsky, A.A., Rudakova, O.A.: Variational problems with pointwise constraints and degeneration in variable domains. Differ. Equ. Appl. 1(4), 517-559 (2009)

21. Kuratowski, K.: Topology, vol. I. Academic Press, New York (1966)

22. Malý, J., Ziemer, W.P.: Fine Regularity of Solutions of Elliptic Partial Differential Equations. AMS, Providence (1997)

23. Murat, F.: Sur l'homogeneisation d'inequations elliptiques du 2ème ordre, relatives au convexe $K\left(\psi_{1}, \psi_{2}\right)=\left\{v \in H_{0}^{1}(\Omega) \mid \psi_{1} \leqslant v \leqslant \psi_{2}\right.$ p. p. dans $\left.\Omega\right\}$. Publ. Laboratoire d'Analyse Numérique, No. 76013, 23 pp. Univ. Paris VI (1976)

24. Rudakova, O.A.: On $\Gamma$-convergence of integral functionals defined on various weighted Sobolev spaces. Ukr. Math. J. 61(1), 121-139 (2009)

25. Vainberg, M.M.: Variational Method and Method of Monotone Operators in the Theory of Nonlinear Equations. Wiley, New York (1973)

26. Zhikov, V.V.: Questions of convergence, duality, and averaging for functionals of the calculus of variations. Math. USSR-Izv. 23(2), 243-276 (1984)

27. Zhikov, V.V.: On passage to the limit in nonlinear variational problems. Russ. Acad. Sci. Sb. Math. 76(2), 427-459 (1993) 